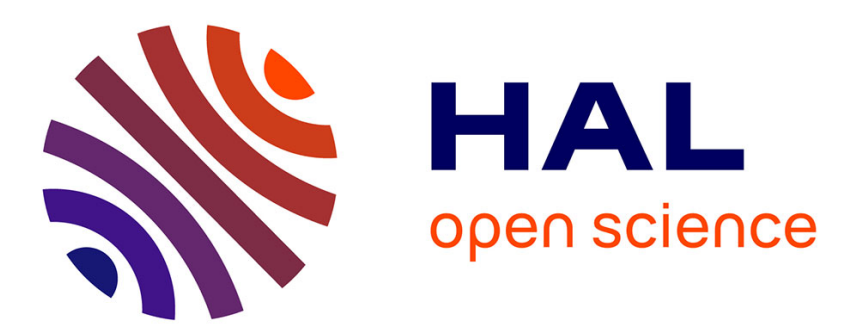

\title{
Les armatures à encoches latérales et à encoches basilaires à la fin du Néolithique, des Alpes à la façade atlantique
}

Maxime Remicourt, Sylvie Saintot, Pierre-Jérôme Rey

\section{- To cite this version:}

Maxime Remicourt, Sylvie Saintot, Pierre-Jérôme Rey. Les armatures à encoches latérales et à encoches basilaires à la fin du Néolithique, des Alpes à la façade atlantique. Bulletin de la Société préhistorique française, 2018, pp.125-147. 10.3406/bspf.2018.14863 . hal-02322806

\section{HAL Id: hal-02322806 \\ https://hal.science/hal-02322806}

Submitted on 27 Jan 2020

HAL is a multi-disciplinary open access archive for the deposit and dissemination of scientific research documents, whether they are published or not. The documents may come from teaching and research institutions in France or abroad, or from public or private research centers.
L'archive ouverte pluridisciplinaire HAL, est destinée au dépôt et à la diffusion de documents scientifiques de niveau recherche, publiés ou non, émanant des établissements d'enseignement et de recherche français ou étrangers, des laboratoires publics ou privés. 


\title{
Les armatures à encoches latérales et à encoches basilaires à la fin du Néolithique, des Alpes à la façade atlantique
}

\author{
Maxime Remicourt, Sylvie Saintot et Pierre-Jérôme Rey
}

Résumé : À la fin du IV millénaire avant notre ère, les armatures à encoches latérales ou à encoches basilaires sont une nouvelle composante des assemblages lithiques. Leur nombre s'accroît jusqu'à atteindre un maximum au cours de la première moitié du $\mathrm{III}^{\mathrm{e}}$ millénaire. Ces artefacts s'inscrivent dans un phénomène global de multiplication des morphotypes d'armatures de flèche à la fin du Néolithique dans le Sud de la France et sur ses marges septentrionales. Les armatures à encoches latérales ou à encoches basilaires ont un temps été considérées comme spécifiques du Néolithique final des bassins du Rhône et de la Saône; conception aujourd'hui révolue car la carte de répartition actualisée montre que leur extension ne se limite pas à cette entité géographique. Leur large diffusion englobe en effet le Sud de la Bourgogne, le Jura méridional, la Suisse occidentale, la Savoie et le Dauphiné. Mais, elles sont bien représentées également en Drôme, en Ardèche et en Charente-Maritime, contrairement à la Provence, au Languedoc oriental et aux Grands Causses où leur diffusion est moindre, et en Italie du Nord, où leur présence semble, pour l'heure, anecdotique. Les plus anciens cas bien datés apparaissent de manière quasi-synchrone en Charente et dans le Jura méridional. Plusieurs modèles d'armatures à encoches se retrouvent de part et d'autre de cette vaste sphère géographique, certains sous-types se cantonnant à des territoires plus restreints. Un modèle particulier, l'armature à encoches basilaires, spécifique à la Charente, se retrouve également en Languedoc, en Provence et dans le Jura, ce qui vient appuyer l'hypothèse de contacts entre ces zones. Contacts qui sont d'ailleurs documentés par d'autres éléments de la culture matérielle. L'éventuel sens préférentiel de ces influences ne peut être encore précisé sur la base des documents archéologiques. Toutefois, la relation que l'on peut proposer avec l'évolution des dispositifs d'emmanchement des poignards en silex pourrait suggérer une influence conceptuelle originaire d'Italie centro-septentrionale. Enfin, le contexte de découverte des armatures à encoches latérales présente des spécificités géographiques. Dans certaines régions, elles paraissent plutôt en lien avec le domaine des vivants alors que, dans d'autres zones, elles semblent réservées aux dotations funéraires.

Mots-clefs : armatures de flèche à encoches latérales, Néolithique final, types et sous-types, diffusion, contact.

Abstract: Notched arrowheads have long been considered as one of the leading fossils of the Late Neolithic in France and Western Switzerland, in the Jura, the Western Alps and the Saône valley. However, the distribution maps of these artefacts prove that they are not limited to this geographical area. An inventory based on both our work and more extensive bibliographic research enabled us to identify 236 points on 142 deposits, and we propose a revision of knowledge concerning this particular artefact. The distribution of notched arrowheads encompasses southern Burgundy, southern Jura, western Switzerland, Savoie and Dauphiné, but also the Drôme, Ardèche and Charente-Maritime. In Provence, in eastern Languedoc and in the Grands Causses their distribution is more limited and in northern Italy their presence is anecdotal.

For the southern zone, the arrowheads discovered in Ardèche, Languedoc, Provence and in the middle Rhone valley in reliable contexts correspond to the interval 2900-2600 BCE. In the Jura and Alpine regions, the oldest lateral notched points are represented in the Clairvaux group from the 31 st century, between 3050 and 2950 BCE. However, most of the Jura and Western Switzerland models belong either to the recent Lüscherz or to the recent Clairvaux, between 2900 and $2700 \mathrm{BCE}$, or to the early phases of the Auvernier-Cordé or to those of the later Chalain group. Lastly, between the Charente and the Gironde estuary, there is a concentration of points with basal notches, which appeared in $3100 \mathrm{BCE}$ in Artenac I contexts for the oldest specimens.

Although many examples are of siliceous raw material of undetermined or indeterminable origin, the few points analysed are often shaped from regional or local raw materials. Therefore, while some of these are imported in the form of finished products, many are produced locally. Several types of notched arrowheads constitute the series. The notched arrowheads are basically round (type 1), or crushed, straight or convex base models, sometimes with spurs or lugs (type 2). Other types are lozenge-shaped (type 3) or lanceolate (type 4) with more or less median notches. Finally, some foliate models with a rounded or acute base are also represented within the lithic assemblages (type 5). Some types seem to be common in the various geographical areas (type 1a), while others have their distribution restricted to small territories (type 2c, for example). According to the general shape of these different models, no morphotype seems directly associated with the presence of notches. 
In general, lozenge-shaped and straight sections dominate. From a chronological point of view, it is difficult to observe a precise succession between the different models. The basal groove points (type 1a) appear in both the Ferrieres and Clairvaux series, but also in the Artenac I series. This type is the most frequent and is also the one most widespread geographically. Subsequently, in the first half of the 3rd millennium BC, arrowheads with lateral notches and basal notches varied in shape (lanceolate, ogival, foliaceous or lozenge-shaped), in their notches (deep or not very marked), or in their base (straight, rounded, squared or escutcheon). Similarly, there are certain peculiarities in the geographical distribution of types. The Artenacian complex shows a clear dominance of type 1a, while the Languedoc and Provençal sectors, found in Ardèche and Drôme, are distinguished by a good representation of types $5 \mathrm{~b}$ and $1 \mathrm{a}$, and are also the only ones to deliver type 2c. Western Switzerland and the Dauphiné / Savoie sector show a dominance of type 5a and more or less similar rates for seven other types. The Southern Jura shows a good representation of type 3a with respect to types 5a, $1 \mathrm{a}$, and even $3 \mathrm{c}$, the latter three being however represented among the Jura models. In the Saône-Rhône group, type 3 is dominant, but types $1 \mathrm{~b}, 2 \mathrm{a}$ and $5 \mathrm{a}$ are also present. The distribution of these elements is therefore restricted to welldefined geographical areas, which reinforces the impression that it is a specific artefact for certain groups.

Morphometrically, the points also exhibit great variability. However, the length / width ratio is generally 2:1, while the criterion of thickness is more random. Unlike the shaping of certain late Neolithic points, no intention of producing a stereotyped form can be clearly established for the manufacture of the notched arrowheads.

Observation of the measurements of the pieces according to the geographical sectors shows, despite the general variability, that the largest pieces inventoried are generally in the south. Overall, their measurements are compatible with efficient use. Most individuals are present in domestic contexts, with 74 deposits for 157 pieces, and 42 individuals delivered in 33 funerary sites. For the eastern specimens, there appears to be a north-south gradient in the status of these pieces, which could be explained in the South of France by a secondary use of these points in deposits and funeral endowments - gestures or intentions rarely or never documented among the elements of the Segonian, Jurassian and circum-Alpine domains. This phenomenon is, moreover, a constant which applies to all the Late Neolithic arrowheads on these territories.

The question of the geographical origin of these elements remains complex because the oldest well-dated cases appear almost synchronously in the Charente and in the southern Jura, which supports the hypothesis of contacts between these zones. Contacts are documented by other categories of material culture. The possible preferential direction of these influences cannot yet be specified on the basis of archaeological documents. However, the relationship that can be proposed with the evolution of flint daggers could suggest a conceptual influence originating in central and northern Italy. Their development takes place in a chrono-cultural sequence which sees the multiplication of types of arrowheads in both the south of France and in the Alpine and Jurassian domains. This multiplication constitutes a specificity of the late Neolithic period in southern France and in western Switzerland. This general phenomenon, which enables the coexistence of up to a dozen morphotypes to be observed, is commonly interpreted as an exacerbation of social competition in a society in full hierarchical mutation, or even as an affirmation of values relating to identity in a warlike context.

Keywords: notched arrowheads, Late Neolithic, types and subtypes, distribution, contact.

$\mathrm{L}$ ES ARMATURES DE FLÈCHE sont considérées par de nombreux chercheurs comme un élément dynamique de la culture matérielle chez les populations préhistoriques (Pétillon et al., 2009). En effet, par rapport à d'autres artefacts lithiques, les caractères morphotechniques de certaines d'entre elles varient rapidement dans le temps et l'espace, ce qui en fait de bons marqueurs pour la définition des chronologies et des cultures archéologiques. Ces variations sont interprétées comme des témoins illustrant des changements économiques, sociétaux ou écologiques, à des fins cynégétiques, voire guerrières ou encore statutaires. C'est en s'inspirant de ces différents critères et définitions que nous avons entrepris de réviser nos connaissances sur un artefact particulier qui est signalé dans la littérature archéologique dès l'aube $\mathrm{du} \mathrm{Xx}^{\mathrm{e}}$ siècle et que nous avons eu l'occasion de rencontrer à maintes reprises sur nos différents terrains d'étude. Les armatures de flèche à encoches latérales ont longtemps été considérées comme l'un des fossiles directeurs de la fin du Néolithique en France et en Suisse occidentale, dans les domaines jurassiens, circumalpin et ségoniens (Thévenot et al., 1976; Pétrequin et al., 1988;
Saintot, 1998). Mais, les cartes de répartition de ces artefacts prouvent au contraire qu'ils ne se limitent pas à cette zone géographique. À partir d'un inventaire, à la fois issu de nos travaux et d'une recherche bibliographique plus large, nous tenterons de proposer une insertion chronologique plus ou moins détaillée de ces éléments, tout en nous interrogeant sur la valeur des différences morphotypologiques qui caractérisent ces armatures. Nous chercherons également des indices liés à l'apparition et à la disparition de ces artefacts, et nous nous questionnerons sur le statut de ces pièces au sein des séries lithiques de la fin du Néolithique marquées par la multiplication des types d'armatures de flèche.

\section{RAPIDE HISTORIQUE}

T a présence récurrente des armatures de flèche à encoches latérales dans les mobiliers lithiques des « palafittes » du Jura et de la Haute-Savoie est signalée dès le début du XX ${ }^{\mathrm{e}}$ siècle (Lebrun, 1908; Le Roux, 1908). 
Ces éléments sont alors considérés comme caractéristiques des stations des rivages lacustres jurassiens durant le Robenhausien récent (Piroutet, 1913). Par la suite, il a été proposé que ces armatures à encoches latérales seraient cantonnées géographiquement au Jura méridional, à la Suisse occidentale et à la Savoie durant le Chalcolithique (Bailloud et Mieg de Boofzeim, 1955). Dans l'article collectif sur la "Civilisation Saône-Rhône " (CSR), ces armatures deviennent l'un des fossiles directeurs de cette dernière, au même titre que les fusaïoles en pierre ou que les armatures polies en roche tenace (Thévenot et al., 1976). Cette entité « culturelle» s'étendait de la Côte-d'Or jusqu'en Isère, avec pour limite occidentale le Rhône et la Saône et pour limite orientale, les Préalpes jusqu'au lac de Bienne. Mais le concept monolithique de CSR est rapidement abandonné (Pétrequin et al., 1988), et remplacé par une série de groupes d'amplitude géographique plus restreinte (Clairvaux et Chalain pour le Jura; Lüscherz et Auvernier-Cordé pour la Suisse occidentale). Dans l'aire delphino-savoyarde, l'existence d'un groupe distinct semble pouvoir être proposée à la lumière d'une série de travaux récents (Bocquet, 2005; Cauliez, 2011; Remicourt, 2015; Rey, 2015; Rey et Marguet, 2016). De «type clef » entrant dans la définition d'une culture archéologique à l'échelle géographique et chronologique, l'armature à encoches devient un « type non-essentiel » commun à plusieurs groupes culturels avec des lien plus ou moins étroits (Pétrequin et al., 1988). Une analyse actualisée de leur répartition (fig. 1) et de leurs évolutions était donc devenue nécessaire.

\section{ATTRIBUTION CHRONOCULTURELLE ET RÉPARTITION GÉOGRAPHIQUE DES ARMATURES À ENCOCHES LATÉRALES}

$\mathrm{P}$ our la zone méridionale, les armatures découvertes en Ardèche dans des contextes fiables renvoient soit à la phase de transition entre le Ferrières et le Fontbouïsse, parfois nommé groupe des Bruyères (2900-2700 avant notre ère), à l'abri-sous-roche de la Vessignée à Saint-

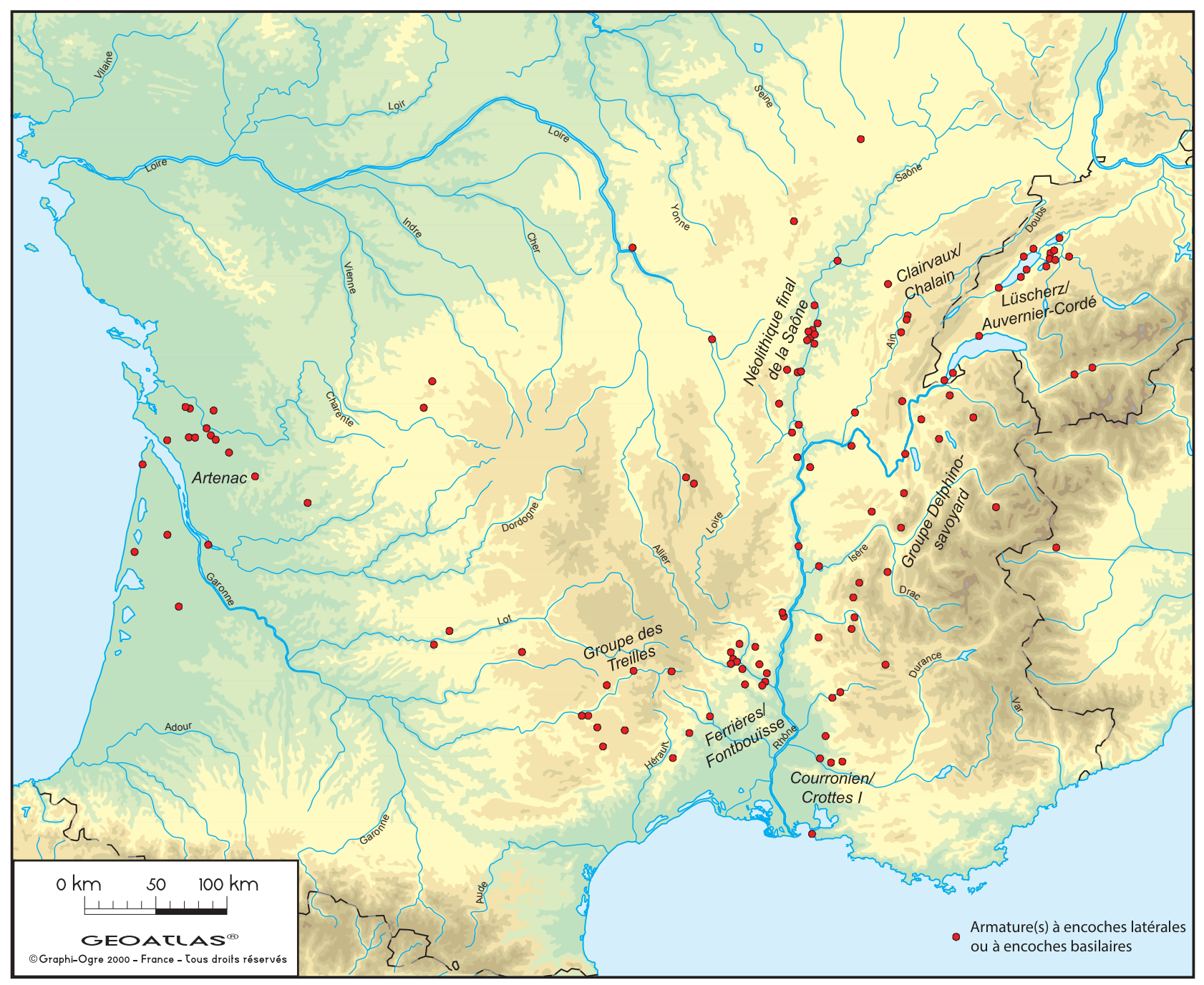

Fig. 1 - Carte de répartition des armatures à encoches latérales et à encoches basilaires.

Fig. 1 -Distribution map of the notched arrowheads. 
Marcel-d'Ardèche (Beaume, 1996), au Fontbouïsse (après 2700 avant notre ère) pour le Rochas, à SaintAlban-Auriolles (Gros, 1999). En Languedoc, quelques pièces appartiennent à une phase presque identique, entre 2900 et 2600 avant notre ère, comme l'illustrent les exemplaires de la grotte de la Rouquette, à Saint-Hilairede-Brethmas, ou à la grotte des Morts, à Durfort, dans le Gard (Remicourt et Bordreuil, 2012). Pour les exemplaires des dolmens du Lamalou et des Feuilles à Rouet dans l'Hérault, la chronologie reste incertaine, mais le mobilier associé s'accorde avec cette tranche chronologique (Arnal et Louis, 1936; Bec Drelon, 2016).

De même, en Provence et dans la moyenne vallée du Rhône, où ces modèles apparaissent dans des ensembles relevant des styles Couronnien ou « Crottes I » (Cauliez, 2011). Trois sites, le Collet Redon et la Couronne, à Martigues dans les Bouches-du-Rhône (Courtin, 1974; Escalon de Fonton, 1977) et l'hypogée du Perpétairi, à Mollans-sur-Ouvèze dans la Drôme (Courtin, 1961), illustrent la présence de ces exemplaires pour cette phase chronologique entre 2900-2600 avant notre ère. Concernant l'armature à encoches de la grotte du Stade à Venasque (Vaucluse), la datation ${ }^{14} \mathrm{C}$ est plus ancienne (MC1480 : $4700 \pm 90 \mathrm{BP}$, soit 3660-3119 avant notre ère à $2 \sigma$ ), mais le mobilier associé renvoie plutôt à une phase récente (Néolithique final II) selon G. Sauzade (1983). Quant aux armatures découvertes dans les dolmens caussenards ou sur des stations de surface, leurs attributions chronologiques s'avèrent très imprécises actuellement, bien que l'on puisse les attribuer au Groupe des Treilles au sens large.

Dans le domaine jurassien et l'arc alpin occidental, les armatures à encoches latérales les plus anciennes sont représentées dans le groupe de Clairvaux à partir du $\mathrm{XXXI}^{\mathrm{e}}$ siècle, entre 3050 et 2950 avant notre ère, dans le Jura (Saintot, 1997). Toutefois, les modèles jurassiens et de Suisse occidentale appartiennent majoritairement, soit au Lüscherz récent ou au Clairvaux récent entre 2900 et 2700 avant notre ère, soit aux premières phases de l'Auvernier-Cordé ou à celles du groupe de Chalain (fig. 2; Saintot, 1998; Honegger, 2001). En Suisse, la présence de ces pièces dans le Lüscherz récent, autour des lacs du Léman, de Neuchâtel et de Bienne, à la Poudrière à Tolochenaz, au parc la Grange à Genève, à Portalban 2 à Delley, à Brise-Lames à Auvernier et à Dorfstation à Lüscherz est également notable. Pour le Jura méridional, les exemplaires de Chalain 2 et 3 à Fontenu et de la Motte-aux-Magnins à Clairvaux-les-Lacs, s'inscrivent dans le Clairvaux récent. Concernant le groupe de Chalain, des exemplaires d'armatures à encoches sont présents dans le Jura parmi deux des gisements précités. En Suisse occidentale, les sites de l'Auvernier-Cordé qui ont livré ces artefacts sont Portalban 2 à Delley, ainsi que Bain des Dames à Saint-Blaise et Avenue-des-Sports à Yverdon. Les armatures à encoches latérales semblent ensuite absentes des séries fiables, après 2550 avant notre ère avec l'arrivée du Campaniforme. Hormis quelques exemplaires anecdotiques qui se rapportent à des datations ${ }^{14} \mathrm{C}$ plus récentes dans le canton de Fribourg à Praz-

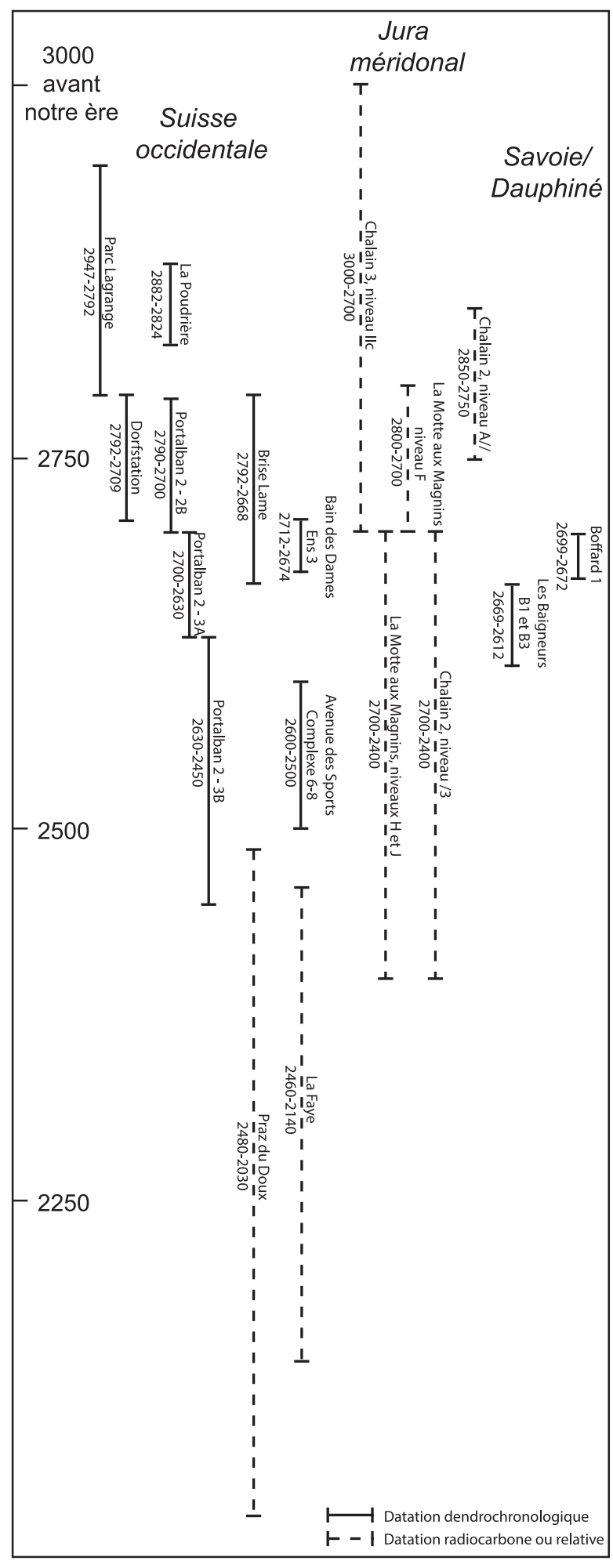

Fig. 2 - Datations dendrochronologiques et ${ }^{14} \mathrm{C}$ de certaines armatures découvertes en Suisse, dans le Jura méridional, en Savoie et en Dauphiné (d'après Saintot 1998; Honegger 2001; Pugin et Corboud 2006; Marguet et Rey 2007; Agustoni et al. 2008; Mauvilly et Ruffieux 2008).

Fig. 2 -Dendrochronological and radiocarbon dates of notched arrowheads discovered in Switzerland, southern Jura, Savoie and Dauphiné (after Saintot 1998; Honegger 2001; Pugin and Corboud 2006; Marguet and Rey 2007; Agustoni et al. 2008; Mauvilly and Ruffieux 2008). 
du-Doux à Frasses-(Ua-11456 : 3835 土 70 BP, soit 24802030 avant notre ère), à la Faye 1 à Lully (ETH-14947 : $3830 \pm 50 \mathrm{BP}$, soit 2460-2140 avant notre ère), et qui pourraient illustrer une perduration de ces éléments dans les phases finales du Néolithique (Mauvilly et Ruffieux, 2008; Agustoni et al., 2008).

Il faut toutefois évoquer, le cas du gisement de Surle-Grand-Pré à Saint-Léonard (Valais), dont l'essentiel des occupations se placent entre 4200 et 3400 avant notre ère et où sont documentées les armatures à encoches latérales en silex, en cristal de roche, en os ou en roche polie $(\mathrm{n}=4$; Winiger, 2009). Cependant l'insertion chronologique de ces éléments reste imprécise car le mobilier des fouilles anciennes est dépourvu de contexte stratigraphique fiable et, par ailleurs, des éléments du Néolithique final sont présents dans les mobiliers issus des fouilles récentes sur le même site (renseignement de P.-J. Rey). Notons qu'une armature à encoches en serpentinite, semblable à celle de Saint-Léonard, a été découverte dans le niveau $5 \mathrm{~b}$ du dolmen MXII de Sion, dont la datation ${ }^{14} \mathrm{C}$ appartient au $\mathrm{III}^{\mathrm{e}}$ millénaire avant notre ère (ETH 4822 : $4055 \pm 65 \mathrm{BP}$, soit 2871-2466 avant notre ère; Favre et Mottet 1990). Enfin, une datation de la fin du Néolithique moyen est également disponible à la Combaz à Chables, dans le canton de Fribourg (Anderson, 2008; Ua-11452 : $4795 \pm 70 \mathrm{BP}$, soit 3710-3370 avant notre ère à $2 \sigma$ ), mais il s'agit probablement d'un charbon hérité, car la céramique découverte avec l'armature est attribuable au Néolithique final.

Le Jura et l'Ouest des Alpes forment une vaste zone géographique qui a fourni les plus grandes concentrations et densités de ce type d'armatures (146 individus sur les 236 recensés). Par exemple, à Charavines (Isère), quatorze armatures proviennent des différents niveaux d'occupations pour six types différents (Gesler, 2005), et trente et une armatures ont été découvertes lors de fouilles récentes et anciennes dans les lacs de Clairvaux et de Chalain (Jura) pour cinq types distincts (Piningre, 1985 ; Saintot, 1998). Au lac d'Aiguebelette (Savoie), différents gisements ont livré une vingtaine d'armatures de flèche pour six types (Rey, 1999; Remicourt, 2015). Enfin en Saône-et-Loire, la fouille du gisement diachronique d'Ouroux-sur-Saône a fourni quinze exemplaires pour six types distincts (Thévenot, 1973).

Un autre ensemble géographique se place entre la Charente et l'estuaire de la Gironde, où l'on observe une concentration d'armatures à encoches basilaires qui apparaît dès 3100 avant notre ère, dans des contextes de l'Artenac I pour les exemplaires les plus anciens (Fouéré, 1994; Fouéré et Dias-Meirinho, 2008). Ainsi, en Charente-Maritime, ces armatures se retrouvent dans la fosse du Peuchin à Pérignac (Fouéré, 1994; GIF9632 : $4460 \pm 50 \mathrm{BP}$, soit 3347-2937 avant notre ère) ou parmi une structure du vallon des Ouchettes à Plassay (Laporte et Marchand, 2002). Quelques pièces plus récentes sont également représentées dans l'Artenac II, comme sur le gisement du Camp, à Challignac en Charente (Fouéré, 1994; Burnez, 2010). Le territoire concerné correspond peu ou prou aux limites dévolues au Peu-Richard mari- time pour la phase chronologique antérieure et les autres zones géographiques d'ambiances artenaciennes ne sont pas concernées par ce phénomène, ou alors de manière anecdotique. Les exemplaires de la vallée du Lot (Clottes, 1964), avec le dolmen de Carbonié, à Saint-Cirq-Lapopie, et celui de la Devèze, à Marcilhac-sur-Célé, pourraient éventuellement trouver un lien avec ces productions. Cette proposition semble également recevable pour les pièces découvertes en Haute-Vienne, au dolmen des Goudours, à Folles, et au dolmen de la Lieue, à Ambazac (Dominique, 1961; Crédot et Dominique, 1973). Néanmoins certains types rappellent les exemplaires orientaux.

Mis à part ces secteurs géographiques, nous constatons l'absence de ces pièces dans la plupart des régions. Ainsi au nord de la Loire, dans le Bassin parisien, elles sont absentes des séries du Seine-Oise-Marne ou du groupe de Gord (Renard, 2003). Dans le quart nordouest, que ce soit en Bretagne ou en Normandie, aucun exemplaire n'est représenté dans les séries lithiques du Néolithique final (Ghesquière et Guyodo, 2008). Elles font également défaut dans le Sud-Ouest, comme dans les ensembles du Vérazien garonnais ou audois (Vaquer, 1990; Briois, 2005). La répartition de ces éléments est donc restreinte à des zones géographiques bien définies, ce qui renforce l'impression qu'il s'agit d'un artefact spécifique à certains groupes. Quelques exemples ethnographiques montrent par ailleurs que la forme de certaines armatures peut être un marqueur culturel fort, comme chez les San du Kalahari où elles appartiennent aux mythes et aux folklores de ces populations; ce qui se traduit par des variations morphologiques des armatures selon les grands groupes linguistiques présents dans ce groupe identitaire, au même titre que les hampes ou les empennages (!Kung, G/wi et!Xo; Wiessner, 1983).

\section{PRINCIPAUX CARACTÈRES TECHNO- TYPOLOGIQUES DES ARMATURES À ENCOCHES LATÉRALES ET RÉPARTITION DES TYPES}

$\mathrm{L}$ a plupart des supports sont indéterminés, en raison du façonnage des pièces généralement par retouches bifaciales couvrantes (fig. 3 et 4), toutefois il est parfois possible de reconnaître quelques lames et éclats, voire du silex en plaquette de Salinelles, comme l'exemplaire du dolmen du Clos de Jacques, à Labeaume en Ardèche (Laforgue et Robert, 1978), ou le silex en plaquette de Collorgues à la grotte des Morts, à Durfort dans le Gard (Remicourt et Bordreuil, 2012). Le silex blond barrémobédoulien vauclusien est, quant à lui, assez bien représenté comme l'illustrent douze armatures retrouvées à la fois dans la région lyonnaise dans le Rhône (Saintot in Motte, 2015), le Jura (Saintot, 1997), en Ardèche (Durand et al., 2005), en Isère, à Charavines (Remicourt, 2016) ou dans le Gard (Remicourt et Bordreuil, 2012). Les armatures à encoches sont fabriquées sur support lamino-lamellaire dans tous les cas et cinq d'entre elles 


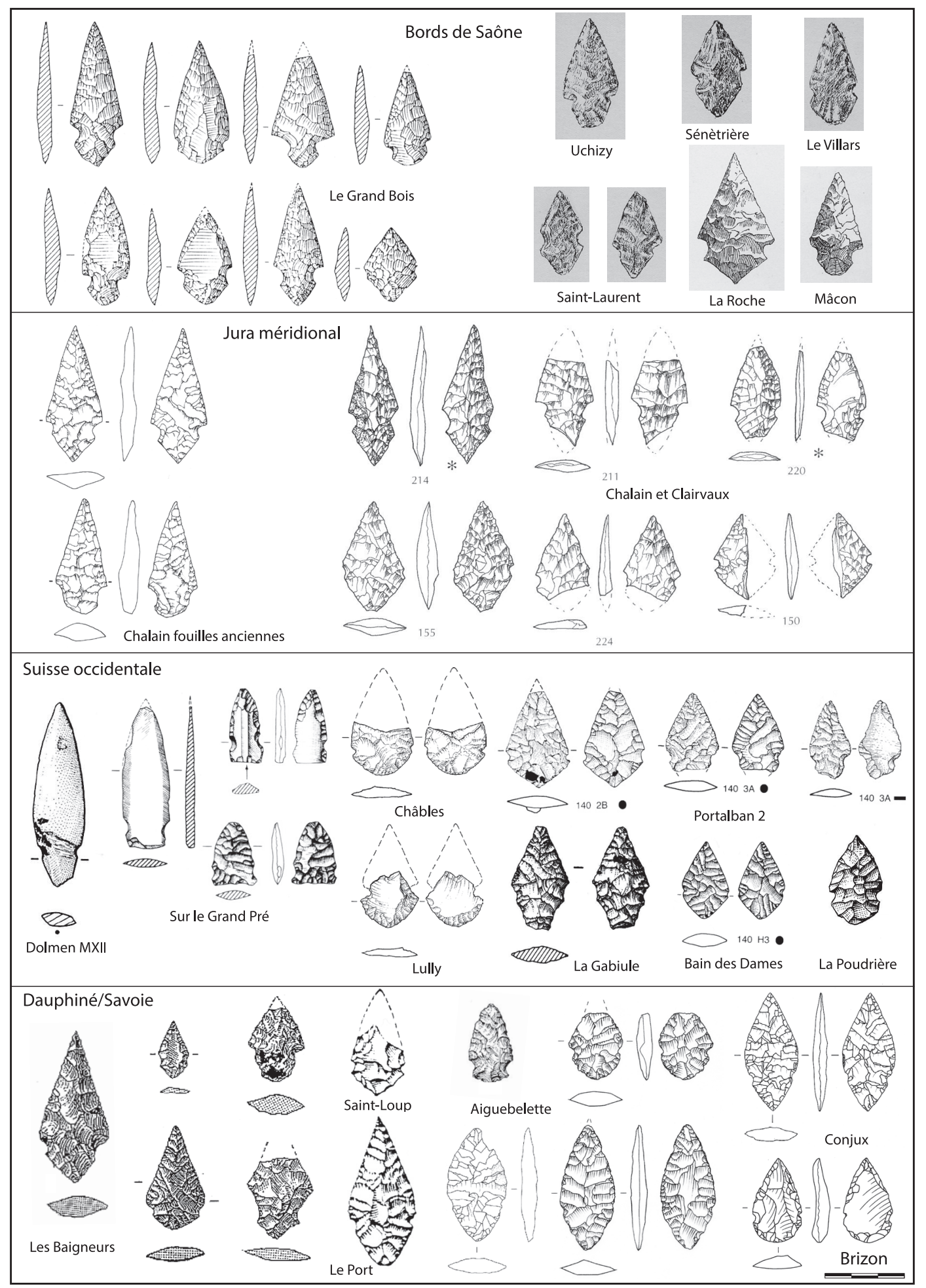

Fig. 3 - Exemples d'armatures à encoches latérales. Bords de Saône : le Grand Bois-le Taillis, Ouroux-sur-Saône, Saône-et-Loire (Thévenot, 1973); la Sénètrière à Sennecé-les-Mâcon, Uchizy, Le Villars, Saône-et-Loire (Lafay, 1932); Saint-Laurent-sur-Saône, Ain (La Fay, 1932); la Roche à Vergisson, Mâcon, Saône-et-Loire (Ferry, 1870). Jura méridional : lac de Chalain, fouilles anciennes, Jura (Piningre, 1985); Chalain 2, Fontenu et la Motte aux Magnins, Clairvaux-les-Lacs, Jura (Saintot, 1998). Suisse occidentale : dolmen MXII, Sion, Valais (Favre et Mottet ,1990); Sur le Grand Pré, Saint-Léonard, Valais (Winiger ,2009); la Faye, Lully, Fribourg (Agustoni et al., 2008); la Combaz, Châbles, Fribourg (Anderson, 2008); Portalban 2, Delley, Fribourg (Honegger, 2001); Bain des Dames, Saint-Blaise, Neuchâtel (Honegger, 2001); la Poudrière, Morges, Vaud (Francillon et Gallay, 1978); la Gabiule, Corsier, Genève (Gallay et Corboud, 1979). Dauphiné-Savoie : les Baigneurs, Charavines, Isère (Gesler, 2005); Saint-Loup, Vif, Isère (Beeching, 1980); le Port, Annecy, Haute-Savoie (Beeching, 1980); Aiguebelette, Savoie (Bocquet, 1976; Remicourt, 2015); les Côtes, Conjux, Savoie (inédit, dessin P.-J. Rey); plateau de Solaison, Brizon, Haute-Savoie (dessin P.-J. Rey; Ginestet, 2001).

Fig. 3 - Examples of notched arrowheads 


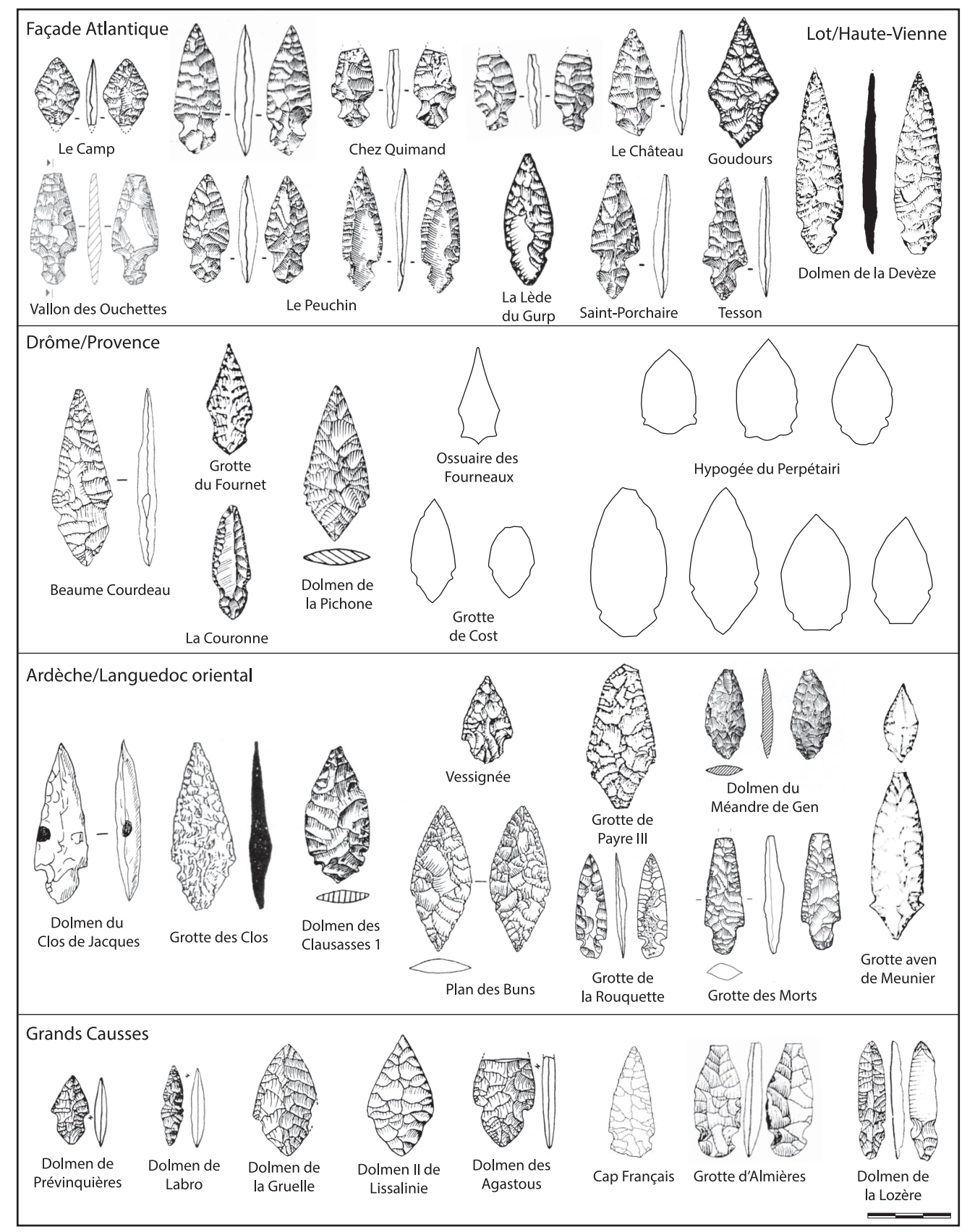

Fig. 4 - Exemples d'armatures à encoches latérales. Façade Atlantique : le Camp, Challignac, Charente (Fouéré et Dias-Meirinho, 2008); vallon des Ouchettes, Plassay, Charente-Maritime (Laporte et Marchand, 2002); Chez Quimand à Ecoyeux, le Peuchin à Pérignac, le Château à Rioux, Tesson, Saint-Porchaire, Charente-Maritime (Fouéré, 1994); la Lède du Gurp, Grayan-et-l'Hôpital, Gironde (Roussot-Larroque, 1984). Massif central : dolmen des Goudours ou Cluzeau, Folles, Haute-Vienne (Crédot et Dominique, 1973), dolmen de la Devèze, Marcilhac-sur-Célé, Lot (Clottes, 1964). Drôme-Provence : Beaume Courdeau, Die, Drôme (Bernard et al., 1987); la Couronne, Martigues, Bouches-du-Rhône (Bailloud et Mieg de Boofzeim, 1955); grotte du Fournet, Montmauren-Diois, Drôme (Beeching, 1980); dolmen de la Pichone, Ménerbes, Vaucluse (Sauzade, 1983); ossuaire des Fourneaux, MoursSaint-Eusèbe, Drôme (d'après Combier, 1977); grotte de Cost, Buis-les-Baronnies, Drôme (d'après Catelan, 1920); hypogée du Perpétairi, Mollans-sur-Ouvèze, Drôme (d'après Courtin,1961). Ardèche-Languedoc oriental : dolmen du Clos de Jacques, Labeaume, Ardèche (Laforgue et Robert, 1978); grotte des Clos au Pouzin et grotte de Payre III à Rompon, Ardèche (Gély, 1993); dolmen des Clausasses 1, Gras, Ardèche (Bonhomme et al., 1967); abri de la Vessignée, Saint-Marcel-d'Ardèche, Ardèche (Beaume, 1996); Plan des Buns, Villeneuve-de-Berg, Ardèche (Saumade, 1991); dolmen du Méandre de Gen, Ruoms, Ardèche (Montjardin, 1974); grotte aven de Meunier, Saint-Marcel-d'Ardèche, Ardèche (Gilles, 1988); grotte de la Rouquette, Saint-Hilaire-de-Brethmas, Gard (Scimia et Varéa, 1996); grotte des Morts, Durfort, Gard (Remicourt et Bordreuil, 2012). Grands Causses : dolmen de Prévinquières et dolmen de la Gruelle à Cornus, dolmen de Labro à Saint-Georges-de-Luzençon, dolmen des Agastous à Saint-Jean-et-Saint-Paul, Aveyron (Azémar, 1989); dolmen II de Lissalinie, Valady, Aveyron (Maury, 1967); cap Français, Pont-de-Montvert, Lozère (Fages et Paris, 2006); grotte d'Almières et dolmen de la Lozère, à Saint-Rome-de-Dolan, Lozère (Simanjuntak, 1998).

Fig. 4 - Examples of notched arrowheads. 
ont subi un traitement thermique préalable à leur transformation, à la manière de ce que l'on observe pour une partie des productions d'armatures de flèches bifaciales, tant dans le Sud de la France que sur la frange Atlantique (Fouéré, 1994; Saintot, 1998; Convertini, 2016). Au moins un exemplaire en silex du Grand-Pessigny est connu à Sutz-Lattrigen dans le canton de Berne (Mallet, 1992). En Suisse occidentale, quatre exemplaires, et peut-être davantage, sont en silex allochtone. Parmi ceux-ci, une armature en silex tertiaire d'Etrelles (HauteSaône) a été identifiée (Honegger, 2001). Des exemplaires en quartz hyalin sont connus en Haute-Savoie, à la grotte $\mathrm{du}$ Parconnaire à Étrembrières (renseignement P.-J. Rey), et dans le Valais (Winiger, 2009). Dans ce canton, des modèles en serpentinite, comme dans le dolmen MXII à Sion (Favre et Mottet, 1990) ou à SaintLéonard (Winiger, 2009), se retrouvent également. Des armatures en os sont également représentées sur ce dernier site, ainsi qu'au Chenet des Pierres à Bozel en Savoie (fouilles P.-J. Rey). Pour les Grands Causses, les productions connues sont réalisées en partie $(n=6)$ à partir de la chaille locale (Azémar, 1989; Simanjuntak, 1998; Fages et Paris, 2006).

Ainsi, même si de nombreux exemplaires sont en matière première siliceuse d'origine non déterminée ou indéterminable, les armatures étudiées sont souvent façonnées à partir de matières premières régionales ou locales, comme dans le Jura (Saintot, 1998) ou dans le Dauphiné et en Savoie (Remicourt, 2015 et 2016), mais également dans le Valais ou sur les Grands Causses (Azémar, 1989; Winiger, 2009). S'il n'est pas possible pour l'heure de faire le prorata entre matières premières importées et matières premières locales dans les supports usités pour la confection des armatures à encoches, il semble toutefois que la majorité soit produite sur place et non importée sous la forme de produits finis. En effet, nous constatons que les différentes matières premières employées, lorsqu'elles n'ont pas une origine locale ou régionale, correspondent à des matériaux couramment importés sous forme de blocs, de lames, de lamelles ou de plaquettes par les différents groupes de la fin du Néolithique (Saintot, 1997 et
1998; Honegger, 2001 et 2006; Remicourt et Vaquer, 2011).

Plusieurs types d'armatures à encoches composent les séries (fig. 5). Il s'agit d'exemplaires à encoches basilaires et à base plus ou moins arrondie (type 1), ou à base écussonnée, droite ou convexe, parfois pourvue d'ergots (type 2). D'autres types sont losangiques (type 3) ou lancéolés (type 4) à encoches plus ou moins médianes. Enfin, certains modèles foliacés à base arrondie ou aiguë sont également représentés au sein des assemblages lithiques (type 5). Certains types semblent courants dans les diverses zones géographiques (type 1a), alors que d'autres voient leur répartition restreinte à de petits territoires (type 2c; fig. 6 et tabl. 1). D'après la forme générale de ces différents modèles, aucun morphotype particulier n'est directement associé à la présence d'encoches. En effet, les différents types à encoches précités (types 1, 3,4 et 5) présentent des caractères communs par rapport aux différents modèles foliacés, losangiques, ogivaux ou allongés connus parmi les séries néolithiques de Suisse occidentale, du Jura méridional, des bords de la Saône et parmi celles du Sud de la France (Courtin, 1974; Gallay, 1977; Beeching, 1980; Saintot, 1998; Honegger, 2001); ceci, tant d'un point de vue morphologique que morphométrique. Seul le type 2, à base en écusson, trouve des pendants dans les séries drômoises ou vauclusiennes avec les armatures, parfois dites « pistilliformes », à bases en écusson, comme sur les gisements de la grotte des Dentales à Cheval-Blanc, de l'hypogée des Crottes à Roaix pour le Vaucluse, ou de l'hypogée du Perpétairi, à Mollans, de la Baume Courdeau à Die, du Pas de Clavel à Clansayes pour la Drôme, mais également en Ardèche au dolmen des Clausasses à Gras (Bonhomme et al., 1967; Beeching, 1980; Sauzade, 1983; Bernard et al., 1987). Concernant les sections, on note une nette domination des losangiques $(\mathrm{n}=151)$, par rapport aux trapézoïdales $(\mathrm{n}=5)$ ou aux triangulaires $(\mathrm{n}=2)$. Les profils sont généralement droits $(n=171)$ et rarement arqués $(n=3)$. Pour ces types d'armatures, l'adjonction des encoches correspond peut-être à des modalités d'emmanchement. Ainsi, pour les modèles de flèches à encoches présents dans les séries néolithiques du Proche-Orient du $\mathrm{IX}^{\mathrm{e}}$ au $\mathrm{V}^{\mathrm{e}}$ millénaire

\begin{tabular}{|c|c|c|c|c|}
\hline $\begin{array}{c}\text { à encoches } \\
\text { basilaires et } \\
\text { base arrondie }\end{array}$ & $\begin{array}{c}\text { à encoches basilaires } \\
\text { et base droite ou convexe } \\
\text { ou en écusson } \\
\text { avec ou sans ergots }\end{array}$ & $\begin{array}{c}\text { losangique à encoches } \\
\text { latérales }\end{array}$ & $\begin{array}{c}\text { lancéolée } \\
\text { à encoches } \\
\text { latérales }\end{array}$ & $\begin{array}{c}\text { à encoches } \\
\text { latérales }\end{array}$ \\
\hline type 1 & type 2 & type 3 & type 4 & type 5 \\
\hline
\end{tabular}

Fig. 5 - Types et sous-types d'armatures à encoches latérales présents dans les séries lithiques de la fin du Néolithique.

Fig. 5 - Types and sub-types of notched arrowheads present in the lithic industry of the later Neolithic. 

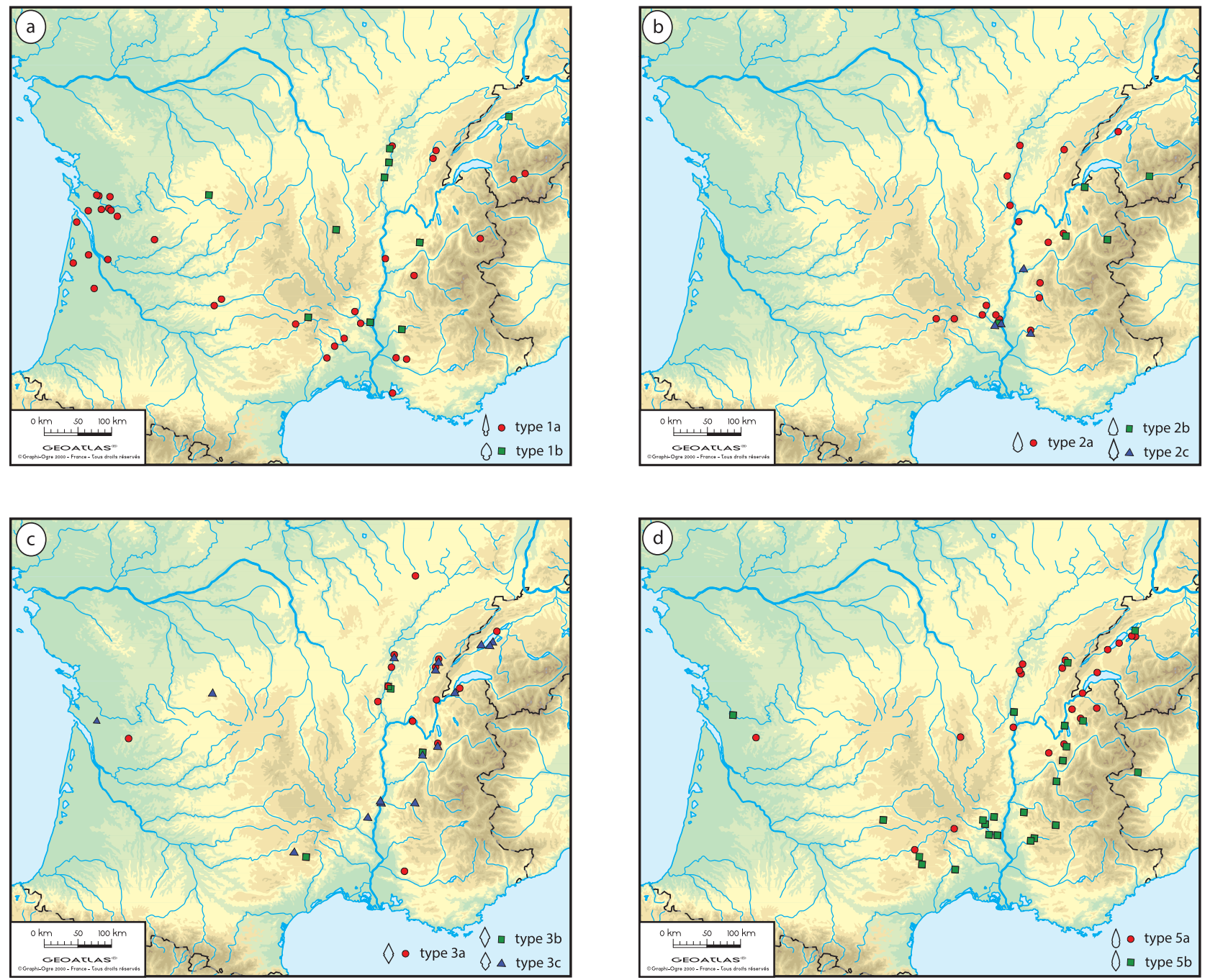

Fig. 6 - Répartition des différents types et sous-types d'armatures à encoches latérales et à encoches basilaires.

Fig. 6 -Distribution map of the various types and sub-types of notched arrowheads.

avant notre ère, il a été proposé qu'elles soient destinées à la mise en place d'une ligature qui les solidariserait à la hampe (Cauvin, 1974). À la manière de ce que l'on connaît pour les productions amérindiennes (corner notched) d'Amérique du Nord (fig. 7; Wilbur, 1990; Callahan, 2001). Hormis cette destination utilitaire, les encoches ne semblent pas devoir accroître le caractère vulnérant de ces pièces, au contraire des armatures à ailerons ou à bords crénelés.

D'un point de vue chronologique, il est difficile de faire ressortir une succession précise entre les différents modèles. Ainsi, les morphotypes les plus archaïques pourraient correspondre aux armatures à encoches latérales opposées et à base droite ou convexe (type 2b) illustrées à Saint-Léonard, dans le Valais (Winiger, 2009), ou au Chenet des Pierres à Bozel en Savoie (fouilles P.-J. Rey) s'il s'avérait qu'elles appartiennent bien à la fin du Néolithique moyen. Les éléments les plus pertinents apparâ̂traient vers la fin du IV e millénaire avant notre ère, avec les armatures à encoches basilaires (type 1a) découvertes dans les séries du Clairvaux (Saintot 1998), de l'Artenac I
(Fouéré, 1994), voire dans celles du Ferrières (Beeching, 1980). Ce type qui est à la fois le plus fréquent (tabl. 1a) est aussi celui que l'on retrouve le plus largement diffusé géographiquement (fig. 6a). Les différents types d'armatures à encoches latérales et à encoches basilaires sont par la suite illustrés durant la première moitié du $\mathrm{III}^{\mathrm{e}}$ millénaire avant notre ère et se diversifient tant au niveau de leur forme (lancéolée, ogivale, foliacée ou losangique) que de leurs encoches (profondes ou peu marquées) ou de leur base (droite, arrondie, équarrie ou en écusson). Par exemple, les stations palafittiques de Chalain, de Clairvaux (Jura) ou celle de Charavines (Isère) illustrent la contemporanéité de différents modèles dans un même niveau d'occupation (Saintot, 1998; Gesler, 2005) et il ne semble pas possible de dégager une trame chronologique précise à partir de critères strictement morphologiques.

Il est possible de cerner la dispersion géographique des types pour faire ressortir des différences au niveau des productions de certains groupes de populations. Si le type 1a domine largement sur la façade atlantique (fig. $6 \mathrm{a}$; tabl. 1a et b), il se retrouve également en Pro- 


a
\begin{tabular}{|l|c|c|c|c|c|c|c|c|c|c|c|c|c|}
\hline Italie du Nord & $1 \mathrm{a}$ & $1 \mathrm{~b}$ & $2 \mathrm{a}$ & $2 \mathrm{~b}$ & $2 \mathrm{c}$ & $3 \mathrm{a}$ & $3 \mathrm{~b}$ & $3 \mathrm{c}$ & 4 & $5 \mathrm{a}$ & $5 \mathrm{~b}$ & Indéterminé & Total \\
\hline Suisse occidentale & 3 & 1 & 1 & 2 & & 2 & & 4 & & 6 & 1 & 6 & 26 \\
\hline Jura méridional & 5 & & 2 & & & 11 & & 4 & 1 & 5 & 1 & 3 & 32 \\
\hline Saône-Rhône & 2 & 5 & 5 & & & 7 & 1 & & & 4 & 1 & 9 & 42 \\
\hline Delphino-savoyard & 2 & 1 & 5 & 4 & & 6 & 1 & 5 & & 13 & 5 & 1 & 43 \\
\hline Drômo-provençal & 6 & 1 & 3 & & 4 & 1 & & 1 & & & 7 & 2 & 25 \\
\hline Languedo-ardéchois & 5 & 1 & 4 & 1 & 2 & & & 3 & & & 7 & 1 & 24 \\
\hline Caussenard & 2 & 1 & 1 & & & & 1 & 1 & 1 & 2 & 3 & & 12 \\
\hline Lot et Haute-Vienne & 2 & 2 & & & & & & 1 & & 1 & & & 6 \\
\hline Façade Atlantique & 19 & & & & & 1 & & 1 & & 1 & 1 & & 23 \\
\hline Total & 46 & 12 & 21 & 8 & 6 & 28 & 3 & 29 & 2 & 32 & 27 & 22 & 236 \\
\hline
\end{tabular}

b
\begin{tabular}{|l|c|c|c|c|c|c|c|c|c|c|c|}
\hline Italie du Nord & $1 \mathrm{a}$ & $1 \mathrm{~b}$ & $2 \mathrm{a}$ & $2 \mathrm{~b}$ & $2 \mathrm{c}$ & $3 \mathrm{a}$ & $3 \mathrm{~b}$ & $3 \mathrm{c}$ & 4 & $5 \mathrm{a}$ & $5 \mathrm{~b}$ \\
\hline Suisse occidentale & 15 & 5 & 5 & 10 & & 10 & & $\mathbf{2 0}$ & & $\mathbf{3 0}$ & 5 \\
\hline Jura méridional & 17,2 & & 6,9 & & & $\mathbf{3 7 , 9}$ & & 13,8 & 3,4 & 172 & 3,4 \\
\hline Saône-Rhône & 6,1 & 15,2 & 15,2 & & & $\mathbf{2 1 , 2}$ & 3 & $\mathbf{2 4 , 2}$ & & 12,1 & 3 \\
\hline Delphino-savoyard & 4,8 & 2,4 & 11,9 & 9,5 & & 14,3 & 2,4 & 11,9 & & $\mathbf{3 0 , 9}$ & 11,9 \\
\hline Drômo-provençal & $\mathbf{2 6 , 2}$ & 4,3 & 13,1 & & 17,4 & 4,3 & & 4,3 & & & $\mathbf{3 0 , 4}$ \\
\hline Languedo-ardéchois & $\mathbf{2 1 , 8}$ & 4,3 & 17,4 & 4,3 & 8,7 & & & 13,1 & & & $\mathbf{3 0 , 4}$ \\
\hline Caussenard & 16,7 & 8,3 & 8,3 & & & & 8,3 & 8,3 & 8,3 & 16,7 & 25 \\
\hline Lot et Haute-Vienne & 33,3 & 33,3 & & & & & & 16,7 & & 16,7 & \\
\hline Façade Atlantique & $\mathbf{8 2 , 8}$ & & & & & 4,3 & & 4,3 & & 4,3 & 4,3 \\
\hline
\end{tabular}

Tabl. 1 - a : tableau de répartition des types et sous-types d'armatures à encoches latérales par secteurs géographiques et en nombres bruts; b : tableau de répartition des types et sous-types d'armatures à encoches latérales par secteurs géographiques et par pourcentage.

Table 1 - a: distribution table of the types and sub-types of notched arrowheads by geographical sectors and numbers; $b$ : distribution table of the types and sub-types of notched arrowheads by geographical sectors and percentage.

vence et en Languedoc, voire jusque dans le Jura méridional, alors qu'il est anecdotique au sein des autres zones géographiques. Le type $1 \mathrm{~b}$ est pour sa part un modèle peu illustré, hormis dans les séries lithiques des bords de Saône. Le type 2 est, quant à lui, plus spécifique

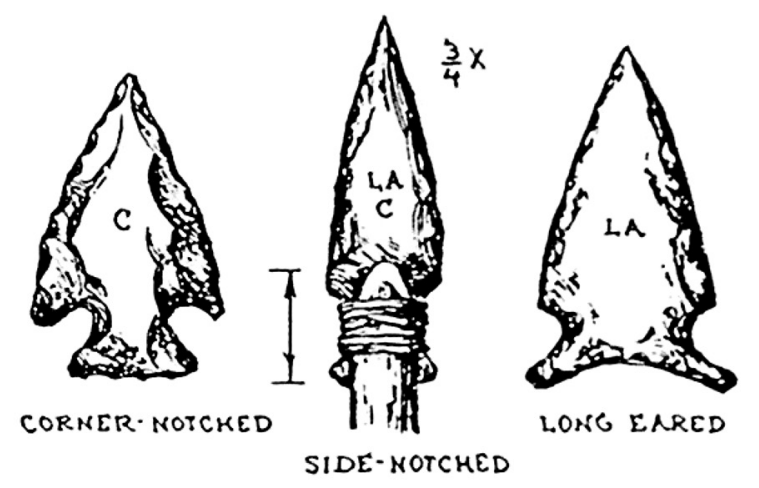

Fig. 7 - Exemples d'armatures à encoches latérales amérindiennes et du système d'emmanchement par ligature (Wilbur, 1990).

Fig. 7 - Examples of Amerindian notched arrowheads and system of hafting by binding (Wilbur, 1990). aux ensembles géographiques orientaux, sans être pour autant un élément courant dans les séries. Nous observons néanmoins certaines spécificités, comme dans le cas du type $2 \mathrm{c}$ qui se cantonne à la moyenne vallée du Rhône, en Ardèche et dans la Drôme (fig. 6b; tabl. 1a) ou de celui $\mathrm{du}$ type $2 \mathrm{~b}$ qui est principalement illustré en Savoie et dans le Valais. Le type 2a est pour sa part plus courant le long de la Saône et du Rhône que dans les autres zones de diffusion (fig. $6 \mathrm{~b}$; tabl. 1a et b). Le type 3 est relativement courant et sa répartition concerne les différents secteurs géographiques à armatures à encoches (fig. 6c). Néanmoins, ce type est bien mieux illustré sur les bords de Saône et dans les séries du Jura méridional que dans celles de Suisse occidentale ou de l'ensemble delphinosavoyard (tabl. 1a et b). Pour la Provence, le Languedoc ou l'Ardèche, mais également la façade atlantique, ce modèle est nettement plus anecdotique. Au niveau des sous-types, le type 3a domine dans le Jura méridional, alors que dans les autres zones, les types $3 \mathrm{a}$ et $3 \mathrm{c}$ sont représentés de façon quasi-équivalente. Le type $3 b$ est quant à lui rarement illustré. Le type 4 n'est reconnu qu'en Aveyron, au dolmen de Labro, à Saint-Georgesde-Luzençon, avec un exemplaire en chaille (Azémar, 1989) et à Chalain 3 dans le Jura (Saintot, 1997). Comme 
le type 3, le type 5 se retrouve dans les différentes zones géographiques (fig. 6d). Néanmoins, nous constatons que le type 5a est le mieux représenté dans les ensembles de Suisse occidentale et de Dauphiné-Savoie, alors que le type $5 \mathrm{~b}$ l'est davantage pour les ensembles des secteurs languedocien, ardéchois, drômois et provençal (tabl. 1a et b). Le type $5 \mathrm{a}$ est également représenté le long de la Saône ou dans le Jura méridional.

Ainsi il est possible de montrer des particularités en lien avec les types et les secteurs géographiques. Le complexe artenacien se singularise par une nette dominance du type $1 \mathrm{a}$, alors que les secteurs languedociens et provençaux, étendus à l'Ardèche et la Drôme, se singularisent par une bonne représentation des types $5 \mathrm{~b}$ et $1 \mathrm{a}$, et ils sont également les seuls à livrer des armatures de type 2c. La Suisse occidentale et le secteur DauphinéSavoie présentent un certain nombre de similarités, avec une dominante d'armatures de type $5 \mathrm{a}$ et des taux plus ou moins proches pour sept autres types d'armatures. Le Jura méridional s'illustre pour sa part avec une bonne représentation du type $3 \mathrm{a}$ par rapport aux types $5 \mathrm{a}, 1 \mathrm{a}$, voire $3 \mathrm{c}$, ces trois derniers étant toutefois représentés parmi les modèles jurassiens. Dans l'ensemble SaôneRhône, le type 3 est dominant, mais les types $1 \mathrm{~b}, 2 \mathrm{a}$ et $5 \mathrm{a}$ n'en sont pas pour autant anecdotiques. Pour les autres zones géographiques, comme l'Italie, les Grands Causses ou pour le reste des éléments, les résultats sont difficiles à analyser en raison de l'indigence numérique du mobilier.

Sur le plan morphométrique, les armatures présentent également une grande variabilité : leur module varie entre 2,1 et $8,2 \mathrm{~cm}$ de long pour une largeur comprise entre 1 et $2,97 \mathrm{~cm}$. Quant à leur épaisseur, elle fluctue entre 0,2 et
$0,8 \mathrm{~cm}$ (fig. 8). À noter toutefois que le rapport entre la longueur et la largeur est généralement du double, alors que le critère de l'épaisseur est plus aléatoire. Contrairement au façonnage de certaines armatures de la fin du Néolithique, aucune intention de produire une forme stéréotypée n'est clairement établie pour la fabrication des armatures à encoches. Comme par exemple, à la fin du Terrinien en Corse (Remicourt, 2014), où les armatures répondent à un critère métrique quasi-récurrent qui correspond à des rapports entre longueur et largeur (2 pour 1), longueur et épaisseur (6 pour 1) et largeur et épaisseur ( 3 pour 1). Toutefois si l'on considère chaque type séparément, il apparaît que le type 1a est plus élancé et qu'il est généralement plus long et moins large que les autres. Les types $3 \mathrm{a}$ et $5 \mathrm{a}$, au contraire, sont souvent plus courts et plus larges que les autres modèles. Du point de vue des épaisseurs qui sont généralement comprises entre 0,4 et $0,8 \mathrm{~cm}$ pour les types 1,3 et 5 , les types 2 ont pour leur part tous une épaisseur inférieure à $0,6 \mathrm{~cm}$. Ces différentes données se retrouvent si l'on observe la mesure moyenne des différents types : 1a $(4,7 \times 1,7 \times 0,55 \mathrm{~cm}) ; 1 \mathrm{~b}(3,8 \times 2,2 \times 0,58 \mathrm{~cm})$; $2 \mathrm{a}(4,1 \times 2,1 \times 0,46 \mathrm{~cm}) ; 2 \mathrm{~b}(3,8 \times 1,9 \times 0,48 \mathrm{~cm}) ;$ $2 \mathrm{c}(3,6 \times 1,7 \times 0,5 \mathrm{~cm}) ; 3 \mathrm{a}(3,4 \times 1,9 \times 0,49 \mathrm{~cm}) ; 3 \mathrm{c}$ $(4,5 \times 2 \times 0,53 \mathrm{~cm}) ; 5 \mathrm{a}(3,3 \times 1,9 \times 0,62 \mathrm{~cm}) ; 5 \mathrm{~b}$ $(4,7 \times 2 \times 0,53 \mathrm{~cm})$. Certains exemplaires se distinguent par leurs mensurations conséquentes, notamment le type $5 \mathrm{~b}$, ou deux pièces allongées font 7,7 et $8,2 \mathrm{~cm}$. Elles proviennent du dolmen de la Lone I, à Saint-Martin-d'Ardèche en Ardèche et de Saint-Pierre-de-Chartreuse en Isère (fig. 9). Un grand exemplaire, de type $3 c$ est également présent à la Beaume Courdeau, à Die (fig. 10). Cette grande taille se retrouve avec l'exemplaire en serpentinite

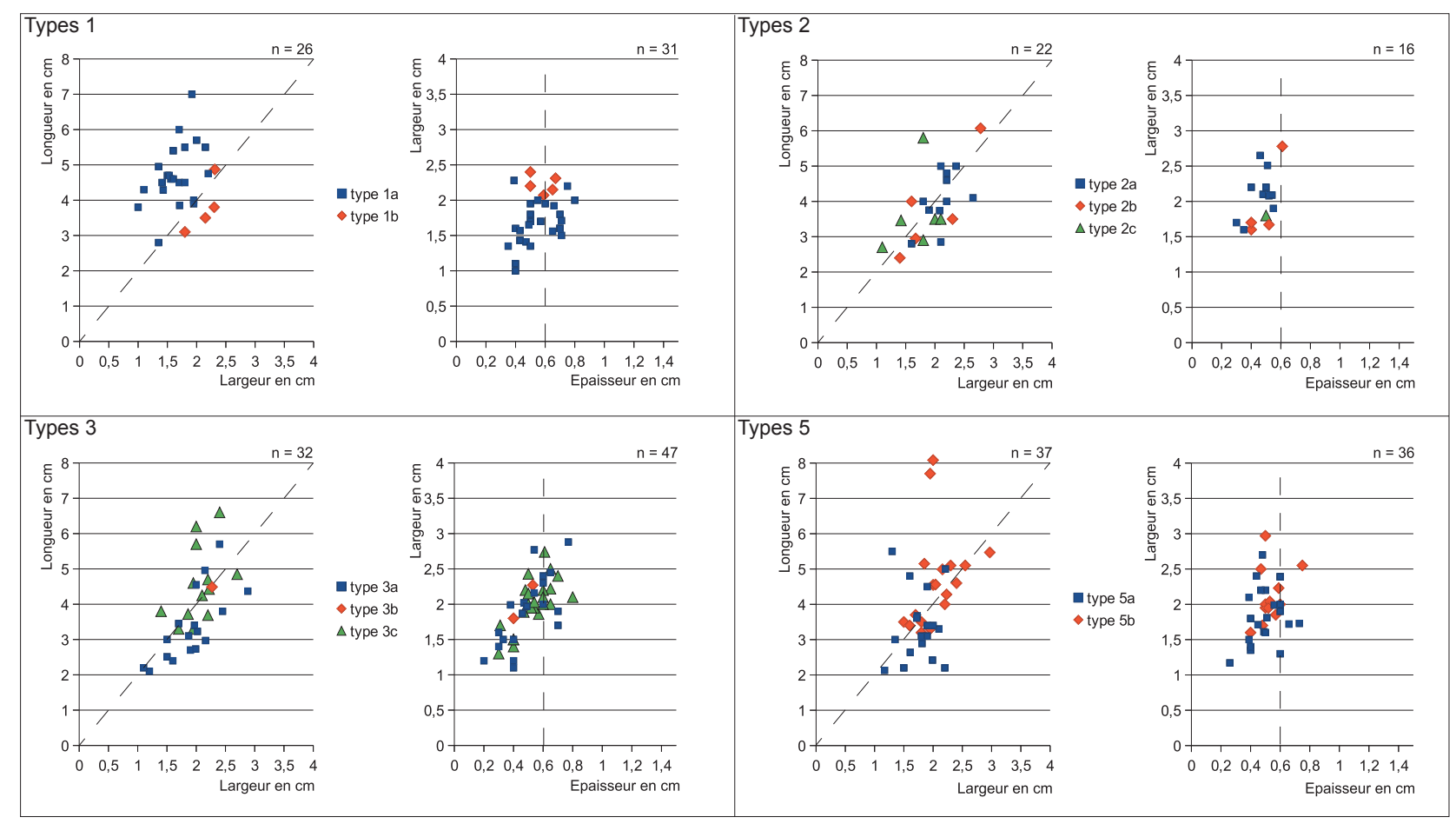

Fig. 8 - Nuages de points des mensurations des types et sous-types d'armatures à encoches latérales.

Fig. 8 -Scatter of points of measurements of the types and sub-types of notched arrowheads. 


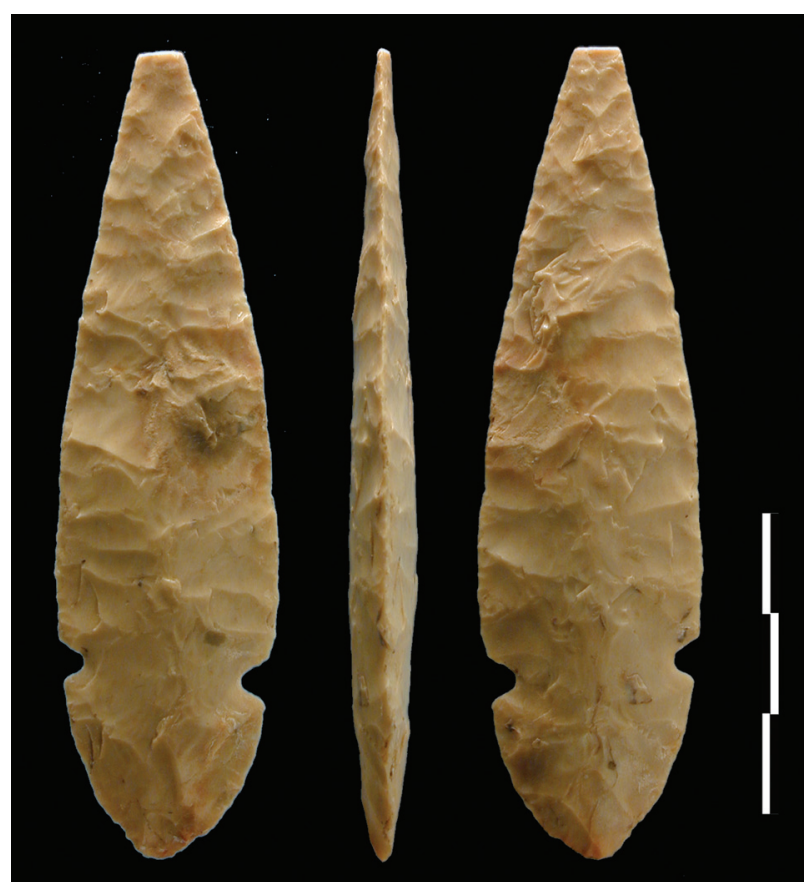

Fig. 9 - Sentier du col de Belfont, Saint-Pierre-de-Chartreuse, Isère (cliché $\mathrm{C}$. Bernard).

Fig. 9 - Path of Col de Belfont, Saint-Pierre-de-Chartreuse, Isère (photo C. Bernard).

de type 1a découvert dans le dolmen MXII à Sion dans le Valais et qui fait $7 \mathrm{~cm}$ de long.

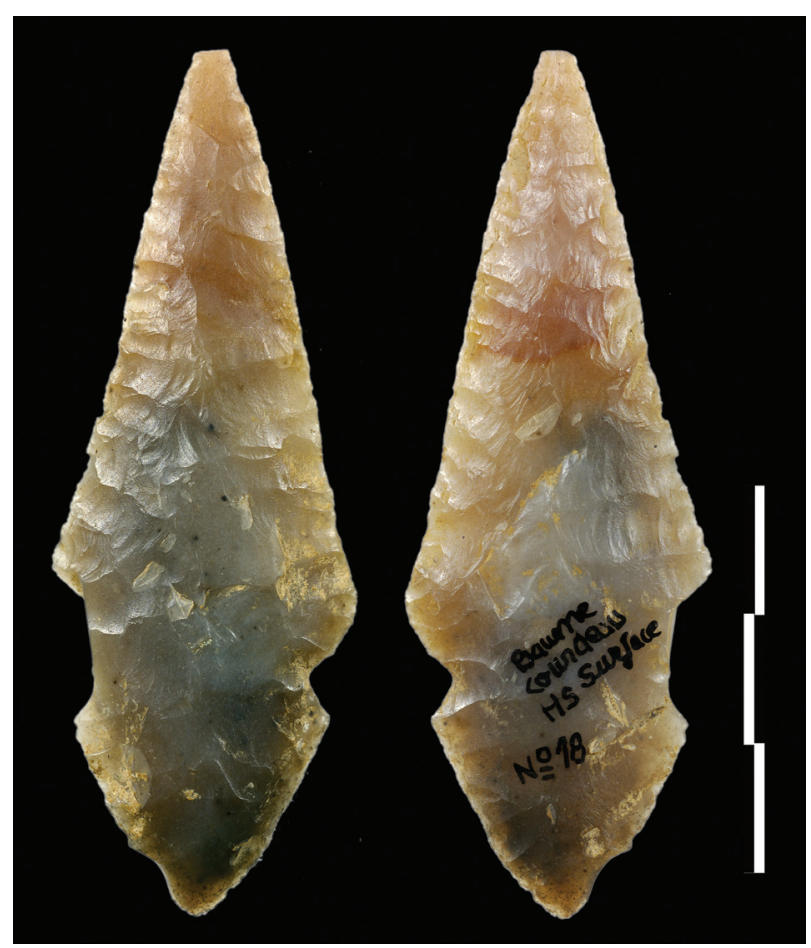

Fig. 10 - Grotte sépulcrale de Beaume Courdeau, Die, Drôme (cliché C. Bernard).

Fig. 10 - Sepulchral cave of Beaume Courdeau, Die, Drôme (photo C. Bernard).
L'observation des mensurations des pièces selon les secteurs géographiques ne semble pas permettre de dégager un schéma simpliste avec des zones à grandes pièces et d'autres à petites. Dans tous les secteurs concernés, les armatures dont les mensurations varient pour un même type coexistent. Seul le type 3 a offre des mensurations peu dispersées, entre 2,1 et $3 \mathrm{~cm}$ de long, sur les gisements de Clairvaux-les-Lacs dans le Jura. On constate néanmoins, que les plus grandes pièces inventoriées sont généralement dans le Sud, notamment dans la Drôme et l'Ardèche, alors que l'Isère et la Savoie sont moins touchées par ce phénomène, et que cette particularité est quasi-absente des gisements des bords de Saône, du Jura méridional et de la Suisse occidentale (hormis dans le cas de matériaux moins usités comme l'os ou la serpentinite).

Bien que l'on observe une certaine variabilité dans les dimensions de ces pièces, leurs mensurations sont compatibles avec une capacité vulnérante efficiente. De fait, il existe une relation entre la largeur et l'épaisseur de l'armature et son efficacité dans le cadre de la chasse au « gros gibier » (Fisher et al., 1984; Fris-Hansen, 1990; Ellis, 1997). Le périmètre minimal pour infliger une blessure mortelle à un cerf étant de $22 \mathrm{~mm}$ et à un aurochs de $26 \mathrm{~mm}$, les diverses armatures à encoches inventoriées se révèlent fonctionnelles pour ce type d'activités. Néanmoins, nous constatons une baisse drastique, dans les séries de la fin du Néolithique, de la faune sauvage pour l'approvisionnement carné des populations préhistoriques; phénomène que l'on identifie depuis le Néolithique moyen (Honegger, 2006; Léa et al., 2009). Si la fonction des armatures de flèche à des fins strictement cynégétiques n'est pas exclusive, leur utilisation courante dans des contextes belliqueux entre hominidés (Guilaine et Zammit, 2001 ; Dias-Meirinho, 2012 ; Vaquer et Bordreuil, 2013) n'est probablement pas non plus leur unique destination.

\section{LES CONTEXTES DE DÉCOUVERTE}

T es contextes de découverte des armatures à encoches correspondent à des habitats, à des ramassages de surface ou à des sépultures, généralement collectives (tabl. 2a; cf. annexe). Nous avons recensé 142 gisements pour 236 armatures, en France, en Suisse occidentale et en Italie du Nord. La majeure partie des individus sont présents dans des contextes d'occupations domestiques, avec septante-quatre gisements pour 157 pièces. La plupart du temps, ces éléments ne sont représentés que par une ou deux pièces, mais certains ensembles en Saôneet-Loire, dans le Jura, en Savoie ou en Dauphiné sont plus forunis. En Saône-et-Loire, la couche d2 de la fosse à ossements du Grand Bois, à Ouroux-sur-Saône, contenait cinq armatures à encoches latérales. Pour le Jura, dans les couches attribuables au groupe de Chalain (2700-2400 avant notre ère), trois exemplaires 
dans le niveau A/ de Chalain 2, à Fontenu ont été recensés (Saintot, 1998). Le même nombre de pièces provient du niveau IIc de Chalain 3, dans une occupation du groupe de Clairvaux (3000-2700 avant notre ère; Saintot, 1997). En Savoie, le site de Boffard 1, à Aiguebelette-le-Lac, a fourni trois exemplaires dans les différents sondages réalisés sur son emprise, pour une occupation datée de 2699-2672 avant notre ère (Marguet, 2003; Remicourt, 2015). En Isère, le gisement des Baigneurs à Charavines a fourni cinq exemplaires dans le niveau $\mathrm{B} 3$, correspondant à l'installation à partir de 2672 avant notre ère, et trois éléments dans le niveau B1, pour l'occupation à partir de 2612 avant notre ère (Gesler, 2005 ; Remicourt, 2016). En CharenteMaritime, le seul gisement qui livre trois exemplaires est celui de Chez Quimand, à Écoyeux, alors que dans la fosse du Peuchin, à Pérignac deux éléments sont présents (Fouéré, 1994). Les autres gisements de la façade atlantique ne fournissent qu'un seul exemplaire en général (Fouéré, 1994).

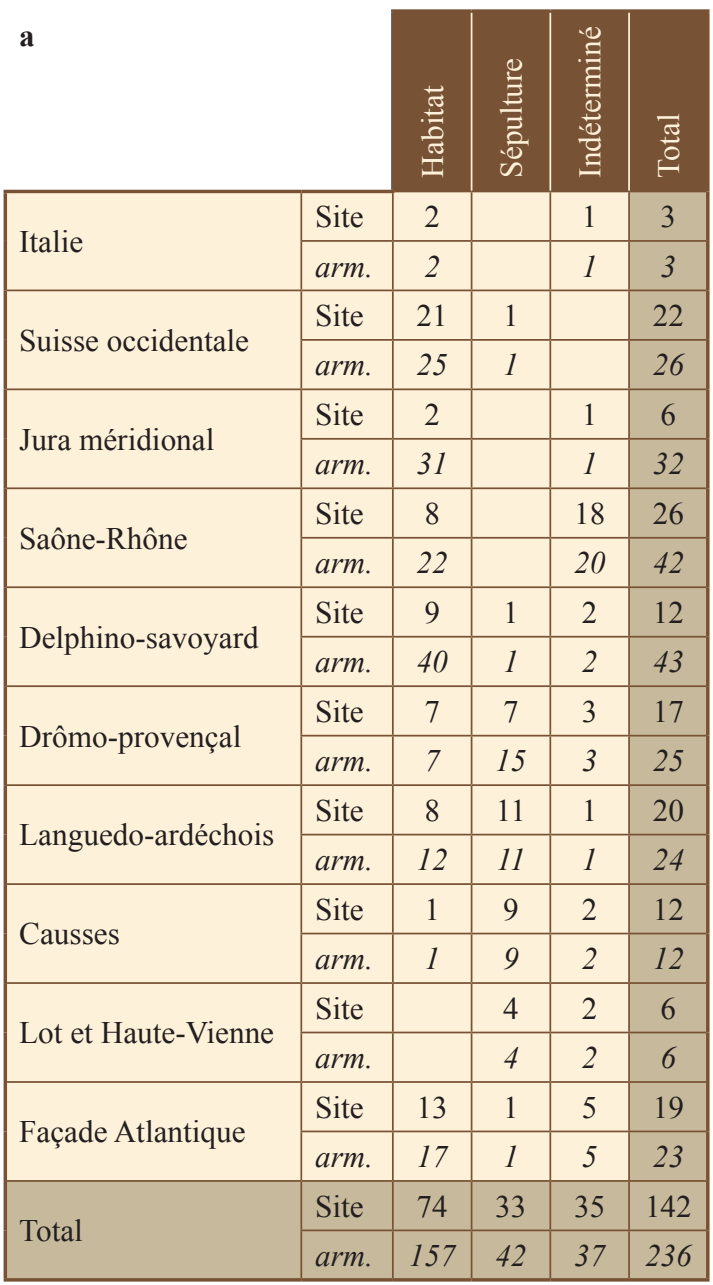

Trente-sept individus, issus de trente-cinq sites à contexte indéterminé, proviennent en grande partie de stations de surface ou de fouilles anciennes. La plupart de ces gisements sont probablement en lien avec les restes d'habitats démantelés.

Pour les contextes sépulcraux, trente-trois gisements ont livré quarante-deux exemplaires. Or, nous observons que, dans certaines zones géographiques, les armatures à encoches ne sont représentées que dans des ensembles sépulcraux, ce qui pourrait indiquer une différence de statut de ces éléments selon les secteurs (tabl. 2b). Ainsi, les quatre exemplaires languedociens sont uniquement présents dans des dolmens ou des grottes sépulcrales. Sur les Grands Causses (Lozère et Aveyron), le schéma est proche avec neuf exemplaires sur douze recensés en lien avec le domaine sépulcral. Pour le Lot et la Haute-Vienne, les quatre armatures inventoriées ont également été découvertes dans des dolmens. Des zones mixtes sont également présentes, comme en Ardèche où l'on recense sept exemplaires

Tabl. 2 - a : tableau de répartition des sites et des armatures à encoches latérales par contextes de découvertes et en nombres bruts; $\mathrm{b}$ : tableau de répartition des sites et des armatures à encoches latérales par contextes de découvertes et par pourcentage.

Table $2-a$ : distribution table of settlements and notched arrowheads by discovery contexts and numbers; $b$ : distribution table of settlements and notched arrowheads by discovery contexts and percentage.

\begin{tabular}{|c|c|c|c|}
\hline \multicolumn{2}{|l|}{ b } & 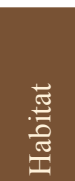 & $\frac{\stackrel{\Xi}{\Xi}}{\frac{0}{0}}$ \\
\hline \multirow{2}{*}{ Italie } & Site & 100 & \\
\hline & arm. & 100 & \\
\hline \multirow{2}{*}{ Suisse occidentale } & Site & 95,5 & 4,5 \\
\hline & arm. & 96,2 & 3,8 \\
\hline \multirow{2}{*}{ Jura méridional } & Site & 100 & \\
\hline & arm. & 100 & \\
\hline \multirow{2}{*}{ Saône-Rhône } & Site & 100 & \\
\hline & arm. & 100 & \\
\hline \multirow{2}{*}{ Delphino-savoyard } & Site & 90 & 10 \\
\hline & arm. & 97,5 & 2,5 \\
\hline \multirow{2}{*}{ Drômo-provençal } & Site & 50 & 50 \\
\hline & arm. & 31,8 & 68,2 \\
\hline \multirow{2}{*}{ Languedo-ardéchois } & Site & 42,1 & 67,9 \\
\hline & arm. & 52,2 & 47,8 \\
\hline \multirow{2}{*}{ Causses } & Site & 10 & 90 \\
\hline & arm. & 10 & 90 \\
\hline \multirow{2}{*}{ Lot et Haute-Vienne } & Site & & 100 \\
\hline & arm. & & 100 \\
\hline \multirow{2}{*}{ Façade Atlantique } & Site & 92,9 & 7,1 \\
\hline & arm. & 94,4 & 5,6 \\
\hline
\end{tabular}


dans les dotations funéraires (dans cinq dolmens et deux grottes sépulcrales) pour dix-neuf éléments recensés. Les douze restantes se distribuant sur huit gisements avec un contexte d'habitat. Le secteur drômo-provençal livre sept gisements funéraires (un hypogée, un dolmen et cinq grottes sépulcrales) pour quinze armatures sur vingt-deux exemplaires recensés avec un contexte de découverte. C'est dans la Drôme que l'on trouve le plus d'armatures à encoches dans un même contexte sépulcral. Il s'agit de l'hypogée du Perpétairi, à Mollanssur-Ouvèze $(\mathrm{n}=7$; Courtin, 1961), ou de la grotte de Cost à Buis-les-Baronnies $(\mathrm{n}=3$; Catelan et Catelan, 1920). Les domaines alpins, jurassiens et ségoniens, de même que la façade atlantique, présentent rarement ce type de mobilier dans des contextes sépulcraux et les armatures semblent cantonnées aux habitats. Pour l'Ouest de la France, l'armature du tumulus de Courcoury en Charente-Maritime constitue un exemple notable (Fouéré, 1994). De même, en Isère, une pièce de ce type a été trouvée dans la sépulture de la Louvaresse à La Balme-les-Grottes (Gély, 1993). Dans le Valais, en Suisse, un exemplaire en serpentinite est présent dans le dolmen MXII à Sion (Favre et Mottet, 1990).

Il est difficile d'interpréter ces différences de localisation entre habitats et sépultures selon les zones géographiques, compte tenu du biais des découvertes archéologiques. Ainsi, dans le Sud de la France, que ce soit en Provence, dans la Drôme, en Ardèche ou en Languedoc oriental, des générations de chercheurs ont entrepris de vider ou fouiller les sépultures collectives prioritairement aux contextes d'habitats, et ceci jusque vers les années 1980. Alors qu'en Suisse occidentale ou dans le Jura méridional, les gisements d'habitats de bords de lacs, dont la conservation est exceptionnelle, ont plus fréquemment été explorés que les domaines sépulcraux. Les habitats des bords de la Saône se situent aussi dans une même dynamique. Néanmoins, avec les progrès de l'archéologie préventive depuis une trentaine d'années, il ressort que les armatures à encoches ne sont pas plus fréquemment illustrées sur les habitats de la fin du Néolithique de la plaine languedocienne ou de la Provence, ou sur les sites funéraires du Jura et de Suisse occidentale. Il est donc envisageable de proposer qu'il existe une évolution dans le statut de ces pièces selon un gradient nord-sud, pour les exemplaires orientaux, qui se traduirait dans le Sud de la France par une utilisation secondaire de ces armatures dans les dépôts et dotations funéraires, gestes ou intentions, peu ou pas documentés parmi les éléments des domaines ségoniens, jurassiens et circum-alpin, où l'on doit toutefois noter que les découvertes d'ensembles sépulcraux sont moins fréquentes. Ce phénomène est d'ailleurs une constante qui s'applique à l'ensemble des armatures de flèche à la fin du Néolithique sur ces différents territoires, la quote-part des armatures découvertes en contexte funéraire dans le Sud de la France étant sans commune mesure avec celles en lien avec le monde des vivants, ce constat s'inversant pour les séries lithiques septentrionales.

\section{LA QUESTION DE L'ORIGINE DES ARMATURES À ENCOCHES ET LA MULTIPLICATION DES MORPHO- TYPES D'ARMATURES À LA FIN DU NÉOLITHIQUE}

$\mathrm{L}$ es premières hypothèses sur l'origine des armatures de flèches à encoches se basaient en grande partie sur les propositions émises par A. Beeching, lequel évoquait une apparition dans le secteur médio-rhodanien, sans doute à partir d'exemplaires découverts à la Baume de Ronze, à Orgnac-l'Aven en Ardèche, dans des niveaux attribués au Ferrières (1980). Une influence du Ferrières a également été proposée pour les exemplaires jurassiens (Pétrequin et al., 1988; Saintot, 1998). Pour la Suisse occidentale, une origine provençale a été suggérée (Honegger, 2001), en raison des découvertes survenues dans des ensembles du Couronnien (Courtin, 1974; Escallon de Fonton, 1977) et de la présence de nombreux exemplaires à l'hypogée du Perpétairi, à Mollans dans la Drôme (Courtin, 1961). Pour la façade atlantique, P. Fouéré a noté la présence d'armatures à encoches en Suisse occidentale et dans le Jura méridional sans toutefois proposer que ces dernières aient influé sur l'émergence des exemplaires charentais (1994).

La question de l'origine géographique de ces éléments reste néanmoins complexe, car les éléments bien datés les plus archaïques restent encore très peu nombreux. Les premiers exemplaires fiables apparaissent quasi-simultanément dans la fosse du Peuchin, à Pérignac en Charente-Maritime, au cours de l'Artenac I (Fouéré, 1994), et dans le Jura, où ils sont signalés à partir de 3050-3010 avant notre ère (Saintot, 1998). En Provence, les exemplaires découverts sur les occupations couronniennes ou les sépultures de style de «Crottes I », se positionnent à partir de 2900 avant notre ère au plus tôt.

Cette concordance chronologique, entre la frange Atlantique et les mondes alpins et jurassiens, pourrait être le fruit de liens qui existeraient dans le cadre d'échanges entre ces communautés éloignées. Ces liens étant illustrés dès le $\mathrm{XXXI}^{\mathrm{e}}$ siècle avant notre ère par les importations de grandes lames en silex tourangeau du Grand-Pressigny, produites sur des nucléus à crêtes antéro-latérales (de type NACAL), en Suisse occidentale et dans le Jura (Ihuel et Pelegrin, 2008; Mallet et al., 2008). Par ailleurs, il a été proposé qu'une influence occidentale soit l'un des vecteurs du développement des scies à encoches dans les domaines jurassiens et alpins, en parallèle à l'arrivée de récipients artenaciens en céramique fine (Pétrequin et al., 1988; Rey et Marguet, 2016). Pour les armatures à encoches, il est difficile de savoir dans quel sens a pu fonctionner leur transfert, et ce au même titre que les scies à encoches qui apparaissent à la fois dans les séries lithiques de l'Artenac II, à partir de 2700-2600 avant notre ère (Fouéré et Dias-Meirinho, 2008), et dans celles du groupe de Chalain et de l'Auvernier-Cordé vers 2750-2700 avant notre ère (Pétrequin et al., 1988; Honegger, 2001); même si ces dernières ont connu un essor tel qu'on les identifie dans une grande partie de l'hexagone, à l'inverse des arma- 
tures, notamment sur des supports en silex du Grand-Pressigny (Mallet et al., 2008), à l'exception notable du Sud de la France (Remicourt et Vaquer, 2011).

La chronologie des premières armatures à encoches et l'absence de mise en œuvre d'une matière spécifique ne permettent pas de savoir dans quel sens a pu fonctionner leur transfert entre la façade atlantique et les marges alpino-jurassiennes. D'un point de vue typologique, quelques pièces lithiques à encoches latérales ont d'ores et déjà été recensées dans des phases chronologiques antérieures à l'apparition des armatures à encoches. En Italie, il s'agit de poignards, comme dans la tombe $97 \mathrm{de}$ la nécropole de Remedello, mais également à Castellaro di Gottolengo, attribués à la phase du Remedello I entre 3400 et 2800 avant notre ère (De Marinis et Pedrotti, 1997; De Marinis, 2013). Le poignard de l'homme de Similaun, daté de la fin du $\mathrm{IV}^{\mathrm{e}}$ millénaire avant notre ère, comporte également des encoches (Egg et Spindler, 1992). De façon à peu près synchrone, on observe la présence de ce type de pièces en Suisse orientale, à Arbon-Bleiche 3 (3384-3370 avant notre ère) ou à Zurich Kanalisationssanierung, couche 3 (3239-3158 avant notre ère) qui sont façonnés sur des silex importés, notamment du Monte Lessini (Honegger, 2002). L'aménagement d'encoches latérales semble constituer une innovation au niveau de l'emmanchement des poignards qui se développe de part et d'autre des Alpes dans les derniers siècles $\mathrm{du} \mathrm{IV}^{\mathrm{e}}$ millénaire avant notre ère.

En parallèle à ce système d'emmanchement, les poignards peuvent présenter des bases arrondies plus larges que la lame, des pédoncules plus ou moins larges et allongés, des bases triangulaires ou trapézoïdales, voire des ergots entre la lame et la base (fig. 11). Cette multiplicité typologique n'étant pas sans rappeler celle que l'on observe pour les productions d'armatures dans le Sud de la France à la fin du Néolithique. Ainsi, les poignards à base arrondie plus large que la lame sont les pendants des armatures de flèche de type Sigottier (Durand, 1999), alors que les poignards à base triangulaire sont proches des armatures losangiques allongées de Provence (Courtin, 1974) ou du Languedoc (Convertini, 2016). Les poignards à ergots trouvent également leur pendant dans les armatures à ergots du Sud de la France, notamment dans la Drôme, au Pas de Clavel à Clansayes (Beeching, 1980) ou dans le Vaucluse, à l'hypogée du Capitaine, à Grillon (Sauzade, 1983). Ces phénomènes d'imitation perceptibles entre ces deux catégories d'artefacts nous incitent à penser que l'adjonction d'encoches latérales sur les armatures pourrait trouver une origine conceptuelle dans les dispositifs d'emmanchement des poignards de Suisse occidentale et d'Italie du Nord.

Bien qu'il reste difficile d'élucider clairement leur région d'origine, leur essor prend place dans une séquence chronoculturelle qui voit la multiplication des types d'armatures à la fois dans le Sud de la France et dans les domaines alpins et jurassiens (Courtin, 1974; Sauzade, 1983 ; Costantini, 1984; Vaquer, 1990; Saintot, 1998; Honegger, 2001 et 2006). Comme dans le cas des armatures à encoches, certains types sont plus fréquents, voire cantonnés, dans certaines entités géographiques données. En prenant l'exemple des armatures à pédoncule très allongé et ailerons plus ou moins dégagés, il apparaît qu'elles sont courantes en Languedoc occidental, en Roussillon et en Catalogne, dans des contextes véraziens (Guilaine et Sacchi, 1977; Vaquer, 1990; Martín et al., 2002; Martín, 2003 ; Briois, 2005). Le cas des «pointes de flèches aveyronnaises » à bords crénelés ou dentelés illustre également ce phénomène. Ces armatures sont très bien documentées dans la région des Grands Causses et sont typiques du Groupe des Treilles, notamment dans sa phase moyenne entre 2800 et 2400 avant notre ère (Costantini, 1984; Vaquer et Bordreuil, 2013); leur dispersion dépasse quelque peu ces frontières culturelles avec quelques artefacts isolés connus jusqu'en

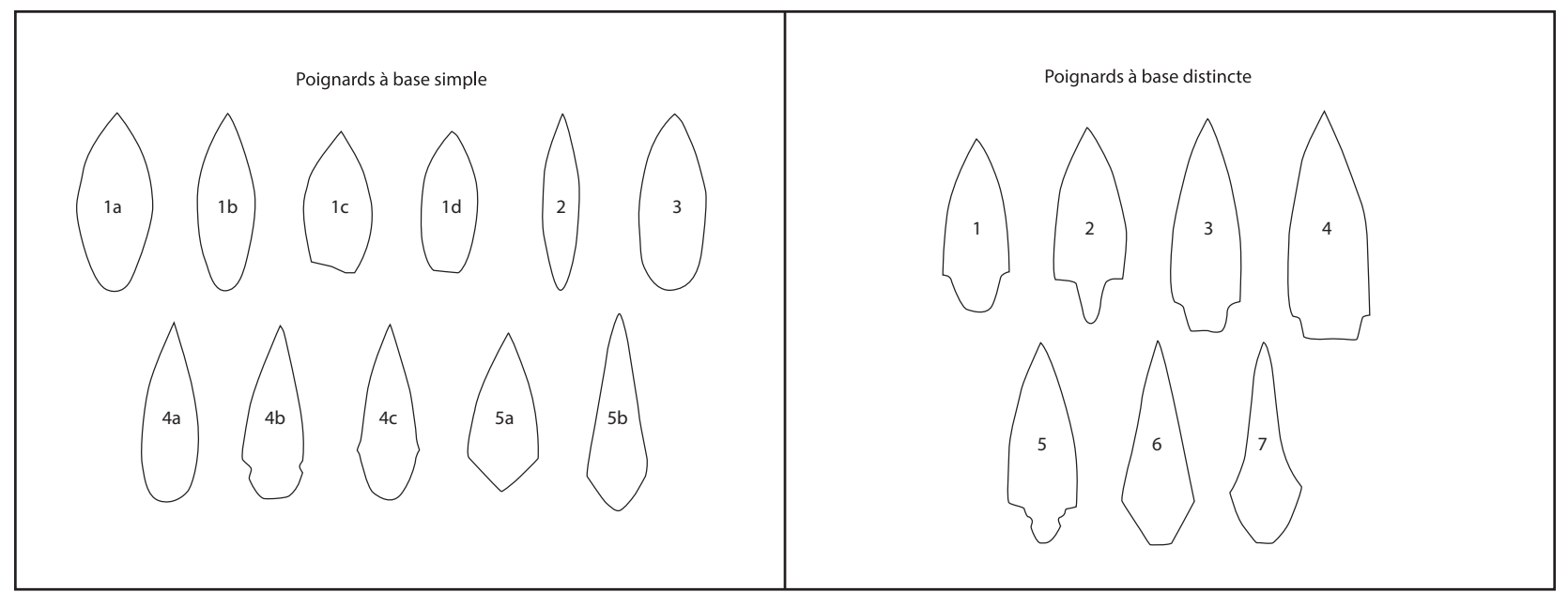

Fig. 11 - Exemple des principales formes de poignards découverts dans les sépultures de la nécropole de Remedello, en Italie (sans échelle, d'après De Marinis et Pedrotti, 1997).

Fig. 11 - Example of the principal shapes of daggers discovered in the burials of the Remedello necropolis, in Italy (no scale, after De Marinis and Pedrotti, 1997). 
Suisse (Honegger, 2001), mais elles restent un marqueur fort des groupes caussenards. Pour les Hautes-Alpes, la Drôme et l'Ardèche, ce rôle de marqueur géographique est tenu par les pointes de Sigottier que l'on peut placer dans un intervalle chronologique de 2900 à 2600 avant notre ère (Sauzade, 1983; Durand, 1999; Bordreuil et al., 2003). Ces armatures à base arrondie large et pointe rétrécie, mises en forme par retouches bifaciales couvrantes, sont principalement découvertes dans des contextes sépulcraux. Comme pour les autres types spécifiques à un secteur géographique, leur dispersion montre que quelques individus sont également présents hors de ces zones (Remicourt et Bordreuil, 2012). Les armatures pistiliformes, qui possèdent parfois des bases en écusson, constituent un autre type spécifique. Possédant un apex affiné, à l'origine de leur nom, ces armatures bifaciales se retrouvent principalement au nord de la Provence, dans la Drôme et en Ardèche (Courtin, 1974; Beeching, 1980; Sauzade ,1983). Cette même répartition semble prévaloir pour les armatures à base en écusson, et parfois à bords concaves, dans les séries de la première moitié du $\mathrm{III}^{\mathrm{e}}$ millénaire avant notre ère (Courtin, 1974; Beeching, 1980; Sauzade, 1983).

Les armatures à encoches latérales ne présentent pas la même répartition relativement restreinte que les exemples précités, hormis dans le cas des armatures à encoches basilaires de l'Artenac. Les exemplaires orientaux semblent plutôt correspondre à ce que l'on observe pour certains types dont la dispersion géographique est beaucoup plus vaste, comme, par exemple, les « pointes de javelot » languedociennes (Audibert, 1962; Arnal, 1963), qui sont des armatures allongées façonnées par retouches bifaciales couvrantes, de forme losangique ou foliacée, dont la longueur est supérieure à $5 \mathrm{~cm}$, et qui sont plus systématiquement illustrées dans les sépultures collectives du bassin méditerranéen, du Roussillon à la Provence, en remontant jusqu'aux Causses (Audibert, 1962; Arnal, 1963; Courtin, 1974; Guilaine et Sacchi, 1977; Sauzade, 1983 ; Costantini, 1984; Azémar, 1989; Vaquer, 1990; Briois, 2005). Ces armatures apparaissent dans les séries d'habitats vers 3100 avant notre ère, notamment dans des ensembles du Ferrières évolué, et perdurent jusqu'à l'arrivée des groupes campaniformes (Convertini, 2016). Si ces pièces ne semblent pas devoir apporter une amélioration significative de la capacité vulnérante d'un point de vue technique, elles représentent un artefact particulier présent à grande échelle, inféodé au bassin méditerranéen et à ses marges, de même que les armatures à encoches orientales, spécifiques à la Saône, au Rhône et à leurs bassins versants, qui sont illustrées dans plusieurs groupes culturels.

Cette multiplication des types d'armatures est une spécificité de la fin du Néolithique en France méridionale et en Suisse occidentale. En effet, au cours du Néolithique moyen, les modèles de type tranchant, pédonculé, foliacé ou losangique varient peu (Saintot, 1998; Honegger, 2001 et 2006; Léa, 2004). Ce phénomène général, qui voit coexister jusqu'à une douzaine de morphotypes, est couramment interprété comme relevant d'une exa- cerbation de la compétition sociale dans une société en pleine mutation hiérarchique (Pétrequin et al., 1988; Saintot, 1998; Honegger, 2006), voire à une affirmation des valeurs identitaires à vocation guerrière (Pétrequin et Pétrequin, 1990; Dias-Meirinho, 2012; Vaquer e t Bordreuil, 2013). Toutefois, les blessures par flèches ne représentant pas une caractéristique courante des défunts de la fin du Néolithique (Guilaine et Zammit, 2001), cette multiplication des types n'est probablement pas un marqueur de l'intensification des conflits entre les groupes. Certains exemples ethnographiques appuient l'idée que ces productions d'armatures de flèche mieux ouvrées dont les types se diversifient pourraient traduire avant tout la valorisation nouvelle de l'armement pour l'affichage de l'identité et du statut social masculin. Chez les Anga de Nouvelle-Guinée (Lemonnier, 1986), par exemple, l'élevage des porcs prime face aux activités cynégétiques, et la diversité des flèches dans le carquois constitue l'un des éléments participant aux rapports de compétition sociale pour atteindre le statut de « Grand Homme ».

\section{POUR CONCLURE}

À la fin du IV millénaire avant notre ère, en France méridionale et en Suisse occidentale, l'apparition des armatures à encoches latérales s'inscrit dans une dynamique qui voit la multiplication des types d'armatures de flèche. Ces pièces constituent le pendant des autres armatures de flèche au niveau de la forme générale, si ce n'est qu'elles sont pourvues d'encoches latérales. C'est durant la première moitié du $\mathrm{III}^{\mathrm{e}}$ millénaire, que ces artefacts sont les mieux documentés et qu'ils se déclinent selon plusieurs modèles. Deux zones de concentrations majeures s'observent, avec d'une part les exemplaires artenaciens de la façade atlantique et, d'autre part, des pièces orientales qui sont principalement localisées le long de la Saône et du Rhône et de leurs bassins versants, de la Suisse à la Méditerranée. Ces modèles à encoches semblent apparaître de manière quasiment synchrone dans ces deux espaces éloignés. Si un développement indépendant reste envisageable, il est plus probable qu'il résulte de liens entre des communautés éloignées, notamment dans le cadre d'échanges de produits finis ou semi-finis; une partie de ces modèles circulant, une autre ayant été façonnée ou imitée localement. Le façonnage des premières armatures à encoches latérales pourrait trouver une origine conceptuelle dans l'évolution des dispositifs d'emmanchement des poignards en silex de Suisse occidentale et d'Italie centro-septentrionale, notamment pour les exemplaires des marges alpines. Ces armatures, qui correspondent majoritairement à des productions locales montrent des variations de morphotypes dominants selon les secteurs géographiques. Nous observons également des différences liées aux contextes de découvertes, les exemplaires méridionaux étant plus fréquents dans les dotations funéraires, alors que les exemplaires septen- 
trionaux ou atlantiques sont majoritairement présents dans des occupations en lien avec l'habitat; ce fait pouvant éventuellement être nuancé en raison d'un déficit de découvertes de contextes sépulcraux dans la partie orientale. À l'instar d'autres artefacts, les armatures à encoches latérales pourraient également traduire le renforcement de la compétition sociale qui semble être inhérente à la fin du Néolithique.

\section{RÉFÉRENCES BIBLIOGRAPHIQUES}

Agustoni C., Castella D., Vigneaud H. (2008) - Lully, la Faye 1-4, in J.-L. Boisaubert, D. Bugnon et M. Mauvilly (dir.), Archéologie et autoroute A1, destins croisés : 25 années de fouilles en terres fribourgeoises, premier bilan (1975-2000), Fribourg, Archéologie fribourgeoise (Archéologie fribourgeoise, 22), p. 253-261.

Anderson T. (2008) - Châbles, la Combaz, in J.-L. Boisaubert, D. Bugnon et M. Mauvilly (dir.), Archéologie et autoroute A1, destins croisés : 25 années de fouilles en terres fribourgeoises, premier bilan (1975-2000), Fribourg, Archéologie fribourgeoise (Archéologie fribourgeoise, 22), p. 272-275.

Arnal J. (1963) - Les dolmens du département de l'Hérault, Paris, PUF (Préhistoire, 15), 250 p.

Arnal J., Louis M. (1936) - La sépulture mégalithique du Lamalou, Cahiers d'histoire et d'archéologie, 11, p. 1-15.

Audibert J. (1962) - La civilisation chalcolithique du Languedoc oriental, Montpellier, IIEL (Monographies préhistoriques et archéologiques, 4), $211 \mathrm{p}$.

AzÉmar R. (1989) - Les mobiliers funéraires des mégalithes $d u$ Larzac aveyronnais, mémoire de l'EHESS, Toulouse, $400 \mathrm{p}$.

Bailloud G., Mieg de Boofzeim P. (1955) - Les civilisations néolithiques de la France dans leur contexte européen, Paris, Picard, 244 p.

Beaume C. (1996) - Le site néolithique final de l'abri de la Vessignée, Saint-Marcel-d'Ardèche, Ardèche archéologie, 13, p. 36-44.

BeEching A. (1980) - Introduction à l'étude des stades Néolithique et Chalcolithique dans le bassin du Rhône moyen, thèse de III ${ }^{\mathrm{e}}$ cycle, université Lyon 2, $673 \mathrm{p}$.

Bec Drelon N. (2016) - Réflexions sur l'implantation et l'architecture des dolmens à couloir et à antichambre du causse de l'Hortus (Hérault, France), in G. Robin, A. D'Anna, A. Schmitt et M. Bailly (dir.), Fonctions, utilisations et représentations de l'espace dans les sépultures monumentales du Néolithique européen, Aix-en-Provence, PUP (Préhistoires de la Méditerranée), p. 35-51.

Bernard E., Billard M., Jourdan A., Lallement J.-L., LAPIERRE G. (1987) - Site funéraire de la Beaume Courdeau, Montagne de Desse, Die-Drôme, rapport de sondage, service régional de l'Archéologie de la région Rhône-Alpes, Lyon, 8 p.

Bocquet A., dir. (2005) - Site néolithique de Charavines, les Baigneurs (Isère, France). Études scientifiques (19722005), Grenoble, Centre de documentation de la Préhistoire alpine, 10 vol., non paginés.

Bonhomme G., Bonhomme C., Arnal J. (1967) - Les dolmens des Clausasses de Gras (Ardèche), Gallia Préhistoire, 10, 2, p. 273-281.
Bordreuil M., Jallot L., Gamberi Almendra de CARvaLho L., Prudhomme F. (2003) - Révision des armatures de flèches de type Sigottier à l'ouest du Rhône, Ardèche archéologie, 20, p. 32-35.

BRIOIS F. (2005) - Les industries en pierre taillée néolithiques en Languedoc occidental, Lattes, ADALR (Monographies d'Archéologie méditerranéenne, 20), 341 p.

Burnez C. (2010) - Le Camp, à Challignac (Charente) au III ${ }^{e}$ millénaire av. J.-C., Oxford, Archaeopress (BAR, International Series 2165), $494 \mathrm{p}$.

Callahan E. (2001) - Artic Archery, Part 3, Bulletin of Primitive Technology, 22, p. 128-133.

Catelan A., Catelan L. (1920) - La grotte sépulcrale de Cost à Buis-les-Baronnies, Bulletin de la Société d'archéologique et de statistique de la Drôme, tome 54, 207 livre, p. 76-88.

CAuliez J. (2011) - Restitution des aires culturelles au Néolithique final dans le sud-est de la France. Dynamiques de formation et d'évolution des styles céramiques, Gallia Préhistoire, 53, p. 85-202.

Cauvin M.-C. (1974) - Flèches à encoches de Syrie : essai de classification et d'interprétation culturelle, Paléorient, 2, 2, p. 311-322.

Clottes J. (1964) - Le dolmen de la Devèze (Marcilhac, Lot), Bulletin de la Société préhistorique française, 61, Études et travaux 2, p. 323-336.

Combier J. (1977) - Rhône-Alpes, Gallia Préhistoire, 20, 2, p. 561-668.

Convertini F. (2016) - Secteur 4, Montpellier, la Cavalade. Du Néolithique moyen à l'Antiquité tardive : occupations néolithiques et Bronze ancien, sépultures néolithiques, fosse et enclos de l'âge du Fer, voie et ensemble funéraire antiques, tome I, vol. 2, rapport final d'opération, INRAP Grand Sud-Est, service régional de l'Archéologie de la région Languedoc-Roussillon, Montpellier, $461 \mathrm{p}$.

Costantini G. (1984) - Le Néolithique et le Chalcolithique des Grands Causses, Gallia Préhistoire, 27, 1, p. 121-210.

Courtin J. (1961) - La sépulture chalcolithique du Perpétairi, Mollans (Drôme), Gallia Préhistoire, 4, 1, p. 192-205.

Courtin J. (1974) - Le Néolithique de la Provence, Paris, Klincksieck (Mémoire de la Société préhistorique française, 11), $335 \mathrm{p}$.

CRédot R., Dominique M. (1973) - Le dolmen des Goudours, commune de Folles (Haute-Vienne), Bulletin de la Société préhistorique française, 70, comptes rendus des séances mensuelles 9, p. 284-288.

Dias-Meirinho M.-H. (2012) - Des Armes et des Hommes. L'archerie à la transition fin du Néolithique-âge du Bronze 
en Europe occidentale, thèse de $\mathrm{III}^{\mathrm{e}}$ cycle, université Toulouse 2 - Le Mirail, 2 vol., 692 p.

Dominique M. (1961) - Le dolmen de la Lieue, commune d'Ambazac (Haute-Vienne), Bulletin de la Société préhistorique française, 58,7 , p. 484-492.

Durand J. (1999) - Les pointes de Sigottier : fait technoculturel et marqueur géographique, in A. Beeching (dir.), Circulations et identités culturelles alpines à la fin de la Préhistoire, Valence, CAP (Travaux du CAP de Valence, 2), p. 231-257.

Durand E., Bigaud P., Franc O. (2005) - Vinezac, La Chapelle-sous-Aubenas, Saint-Sernin, Saint-Etienne-de-Fontbellon, RD 104, déviation de la Chapelle-sous-Aubenas, rapport de diagnostic, INRAP Rhône-Alpes Auvergne, service régional de l'Archéologie de la région Rhône-Alpes, Lyon, $38 \mathrm{p}$.

EGG M., SPINDler K. (1992) - Die Gletschermumie vom Ende der Steinzet aus den ötzaler Alpen, Jahrbuch des RömischGermanischen Zentralmuseums Mainz, 39, 1, p. 3-128.

ElLIS C. J. (1997) - Factors Influencing the Use of Stone Projectile Tips: An Ethnographic Perspective, in H. Knecht (dir.), Projectile Technology, New York, Plenum Press, p. 37-78.

EsCALON DE Fonton M. (1977) - Le village Néolithique de la Couronne à Martigues (Bouches-du-Rhône), Congrès préhistorique de France, compte rendu de la vingtième session (Provence, 1-7 juillet 1974), Paris, SPF, p. 130-136.

Fischer A., Vemming Hansen P., Rasmussen P. (1984) - Macro and Microwear Traces on Lithic Projectile Points: Experimental Results and Prehistoric Examples, Journal of Danish archaeology, 3, p. 19-46.

FAgES G., PARIS H. (2006) - La montagne du Lozère : traces d'une fréquentation Néolithique, Bulletin $d u$ Centre d'études de recherches littéraires et scientifiques de Mende, 26, p. 3-26.

Favre S., Mottet M. (1990) - Le site du Petit-Chasseur III à Sion (VS). MXII, un dolmen à soubassement triangulaire du début du III millénaire, Archéologie suisse, 13, p. 114-123.

Ferry H. de (1870) - Le Mâconnais préhistorique, Mâcon, Durand, $136 \mathrm{p}$.

Fouré P. (1994) - Les industries en silex entre Néolithique moyen et Campaniforme dans le Nord du bassin Aquitain, thèse de III ${ }^{\mathrm{e}}$ cycle, université Bordeaux I, 2 vol., 551 p.

Fouéré P., Dias-Meirinho M.-H. (2008) - Les industries lithiques taillées des $\mathrm{IV}^{\mathrm{e}}$ et $\mathrm{III}^{\mathrm{e}}$ millénaires dans le Centre-Ouest et le Sud-Ouest de la France, in M.-H. DiasMeirinho, V. Léa, K. Gernigon, P. Fouéré, F. Briois et M. Bailly (dir.), Les industries lithiques $d u I V^{e}$ et III ${ }^{e}$ millénaires en Europe occidentale, Oxford, Archaeopress (BAR, International Series 1884), p. 231-258.

Francillon F., Gallay A. (1978) - Fouille sub-aquatique de sauvetage sur la station lacustre de Morges, la Poudrière, Archéologie Suisse, 1, p. 55-57.

Fris-Hansen J. (1990) - Mesolithic Cutting Arrows: Functional Analysis of Arrows Used in the Hunting of Large Game, Antiquity, 64, 244, p. 494-504.
Gallay A. (1977) - Le Néolithique moyen du Jura et des plaines de la Saône. Contribution à l'étude des relations Chassey-Cortaillod-Michelsberg, Genève, SSPA (Antiqua, 6), $344 \mathrm{p}$.

Gallay A., Corboud P. (1979) - Les stations préhistoriques littorales du Léman : où en sont nos connaissances?, Archéologie Suisse, 2, p. 44-49.

Gely B. (1993) - Les Pratiques funéraires préhistoriques. Inventaire et analyse de sépultures de la région RhôneAlpes, thèse de $\mathrm{III}^{\mathrm{e}}$ cycle, École pratique des hautes études, Paris, 1 vol.

GesLer A. (2005) - Les villages néolithiques de Charavines. Le silex, Grenoble, CDPA, 277 p.

Ghesquiere E., Guyodo J.-N. (2008) - Les industries lithiques taillées des $\mathrm{IV}^{\mathrm{e}}$ et $\mathrm{III}^{\mathrm{e}}$ millénaires dans le quart nord-ouest de la France, in M.-H. Dias-Meirinho, V. Léa, K. Gernigon, P. Fouéré, F. Briois et M. Bailly (dir.), Les industries lithiques du IVe et III ${ }^{e}$ millénaires en Europe occidentale, Oxford, Archaeopress (BAR, International Series 1884), p. 113-133.

Gilles R. (1988) - Grotte-Aven de Meunier, Saint-Martind'Ardèche, Bulletin de la Société préhistorique française, 85, 4, p. 110-114.

Ginestet J.-P. (2001) - Découvertes fortuites, Revue savoisienne, 141, p. 63-64.

Gros O., Gros C.-A. (1999) - Les habitats et les mégalithes de la rive gauche du Chassezac, Ardèche archéologie, 16, p. 7-34.

Guilaine J., Sacchi D. (1977) - Hommage à Philippe Héléna, Cahiers ligures de Préhistoire et d'archéologie, 25-26, $350 \mathrm{p}$.

Guilaine J., Zammit J. (2001) - Le Sentier de la guerre : visages de la violence préhistorique, Paris, Seuil, 371 p.

Honegger M. (2001) - L'industrie lithique taillée du Néolithique moyen et final de Suisse, Paris, CNRS (Monographie du Centre de recherches archéologiques, 24), 198 p.

Honegger M. (2002) - Les influences méridionales dans les industries lithiques du Néolithique suisse, in Les industries lithiques taillées holocènes du bassin Rhodanien, Montagnac, Monique Mergoil (Préhistoires, 8), p. 135-148.

Honegger M. (2006) - Flèches simples et flèches complexes dans le Néolithique du Nord des Alpes, in L. Astruc, F. Bon, V. Léa, P.-Y. Milcent et S. Phillibert (dir.), Normes techniques et pratiques sociales : de la simplicité des outillages pré- et protohistoriques, actes des $26^{\mathrm{e}}$ Rencontres internationales d'archéologie et d'histoire (Antibes, 20-22 octobre 2005), Juan-les-Pins, APDCA, p. 255-266.

Ihuel E., Pelegrin J. (2008) - Du Jura au Poitou en passant par le Grand-Pressigny : une méthode de taille et des poignards particuliers vers 3000 av. J.-C., in M.-H. DiasMeirinho, V. Léa, K. Gernigon, P. Fouéré, F. Briois et M. Bailly (dir.), Les industries lithiques du IVe et III millénaires en Europe occidentale, Oxford, Archaeopress (BAR, International Series 1884), p. 135-182.

LAFAY G. (1932) - Album préhistorique du Mâconnais, Mâcon, Renaudier, $42 \mathrm{p}$. 
LAforgue M., Robert J. (1978) - Premiers résultats de la fouille du dolmen du Clos de Jacques à Labeaume, Ardèche, Société d'études et de recherches archéologiques et historiques de Vagnas (Ardèche), 12, p. 16-23.

Laporte L., Marchand G. (2002) - Une courte occupation artenacienne au Néolithique final, Gallia Préhistoire, 44, p. 86-101.

LÉA V. (2004) - Les industries lithiques du Chasséen en Languedoc oriental, Oxford, Archaeopress (BAR, International Series 1232), $210 \mathrm{p}$.

LÉa V., Gassin B., Linton J. (2009) - Quelles armatures de projectiles pour le Midi méditerranéen et ses marges du milieu du $V^{\mathrm{e}}$ au milieu du IV millénaire?, Gallia Préhistoire, 51, p. 155-177.

Lebrun L. (1908) - Note complémentaire sur quelques pointes à crans latéraux, Mémoires de la Société d'émulation du Jura, $8^{\mathrm{e}}$ série, p. 65-71.

Lemonnier P. (1986) - Le sens des flèches. Culture matérielle et identité ethnique chez les Anga de Nouvelle-Guinée, in De la voûte céleste au terroir, du jardin au foyer, Paris, EHESS, p. 573-595.

Le Roux M. (1908) - La palafitte Néolithique du lac d'Annecy, Congrès préhistorique de France, compte rendu de la quatrième session (Chambéry, 1908), Paris, Schleicher Frères, p. $547-566$.

Mallet N. (1992) - Le Grand Pressigny. Ses relations avec la Civilisation Saône-Rhône, Tours, CTHS (Supplément au Bulletin de la Société des amis du musée du GrandPressigny), $228 \mathrm{p}$.

Mallet N., Ihuel E., Verjux C. (2008) - La diffusion des silex du Grand-Pressigny au sein des groupes culturels des $\mathrm{IV}^{\mathrm{e}}$ et $\mathrm{III}^{\mathrm{e}}$ millénaires avant J.-C., in M.-H. Dias-Meirinho, V. Léa, K. Gernigon, P. Fouéré, F. Briois et M. Bailly (dir.), Les industries lithiques du IV et III ${ }^{e}$ millénaires en Europe occidentale, Oxford, Archaeopress (BAR, International Series 1884), p. 183-206.

Marguet A. (2003) - Élaboration de la carte archéologique des gisements du lac d'Aiguebelette, Bilan scientifique $d u$ DRASSM, 1998, p. 96-110.

Marguet A., Rey P.-J. (2007) - Le Néolithique dans les lacs alpins français : un catalogue réactualisé, in $\mathrm{M}$. Besse (dir.), Sociétés néolithiques, des faits archéologiques aux fonctionnements socioéconomiques, actes du colloque interrégional sur le Néolithique (Neuchâtel, $1^{\text {er }}$ et 2 octobre 2005), Lausanne, CAR (Cahiers d'archéologie romande, 108), p. 335-362.

De Marinis R. C. (2013) - Le necropoli di Remedello Sotto, Volongo e Cumarola : nuovi aggiornamenti, in Le manifestazioni del sacro e l'eta del Rame nella regione alpina e nella pianura padana, actes du congrès, Brescia, Palazzo Broletto, p. 193-222.

De Marinis R. C., Pedrotti A. L. (1997) - L'Eta del Rame nel versante italiano délie Alpi Centro-Occidentali. 3. La Cultura di Remedello: nuovo proposte di cronologica relativa e assoluta, in La valle d'Aosta nel quadro della Preistoria e Protostoria dell'arco alpino centro-occidentale, actes de la $\mathrm{XXXI}^{\mathrm{e}}$ réunion scentifique (Courmayeur, 2-5 juin
1994), Florence, Istituto italiano di Preitoria e Protostoria, p. 260-300.

Martín A. (2003) - Els grups del neolitic final, calcolitic i bronze antic. El inicis de la metal-lurgia, Cota Zero, 18, p. 76-105.

Martín A., Petit M., Maya J. L. (2002) - Cultura material, economia i intercanvis durant el III mil·lenni a Catalunya, XII col-loqui Internacional d'Arqueologia de Puigcerdà, actes du colloque (Puigcerdà 2000), Puigcerdà, IEC, p. 295323.

Mauvilly M., Ruffieux M. (2008) - Frasses, Praz au Doux, in J.-L. Boisaubert, D. Bugnon et M. Mauvilly (dir.), Archéologie et autoroute A1, destins croisés : 25 années de fouilles en terres fribourgeoises, premier bilan (1975-2000), Fribourg, Archéologie fribourgeoise (Archéologie fribourgeoise, 22), p. 204-211.

Maury J. (1967) - Le dolmen II de Lissalinie. Commune de Valady (Aveyron), Bulletin de la Société préhistorique française, 64, 5, p. 151-154.

Montjardin R. (1974) - Le dolmen du Méandre de Gen, commune de Ruoms (Ardèche), Études préhistoriques, 10-11, p. 1-15.

Motтe S. (2015) - Déviation A46 - Quincieux, Rhône, rapport final d'opération, INRAP Rhône-Alpes Auvergne, service régional de l'archéologie de la région Rhône-Alpes, Lyon.

Pétillon J.-M., Dias-Meirinho M.-H., Cattelain P., Honegger M., Normand C., Valdeyron N. (2009) Éclairages actuels sur quelques armatures de projectiles paléo-, méso- et néolithiques, in Recherches sur les armatures de projectiles du Paléolithique supérieur au Néolithique (Palethnologie, 1), p. 1-6.

Pétrequin P., Pétrequin A.-M., Chastel J., Giligny F., SaIntot S. (1988) - Réinterprétation de la Civilisation Saône-Rhône, Gallia Préhistoire, 30, p. 1-89.

Pétrequin P., Pétrequin A.-M. (1990) - Flèches de guerre, le cas des Danis de l'Irian Jaya, Indonésie, Bulletin de la Société préhistorique française, 87, p. 484-511.

Piningre J.-F. (1985) - Les industries lithiques taillées de Chalain et Clairvaux. Collections des musées de Lonsle-Saunier, Dijon et Dole, in Présentation des collections du musée de Lons-le-Saunier, 1. Néolithique ChalainClairvaux, fouilles anciennes, Lons-le-Saunier, musée d'Archéologie, p. 145-167.

Piroutet M. (1913) - Sur la coexistence des populations différentes en Franche-Comté pendant les temps pré- et protohistoriques, Congrès préhistorique de France, compte rendu de la $9^{\mathrm{e}}$ session (Lons-le-Saunier, 1913), Paris, Schleicher Frères, p. 560-652.

Pugin C., Corboud P. (2006) - Un habitat littoral du Néolithique final en bordure de Genève, Annuaire d'archéologie suisse, 89, p. 25-50.

Remicourt M. (2014) - Étude de l'industrie lithique taillée de la couche B, terrasse nord, à I Calanchi (Sollacaro, Corse$d u$-Sud), rapport d'études, service régional de l'Archéologie de la région Corse, Ajaccio $154 \mathrm{p}$.

Remicourt M. (2015) - Étude des industries lithiques taillées du lac d'Aiguebelette en Savoie: Beau Phare 1, le Gojat, 
Petite Île, Grande Île et les Roseaux, rapport d'études, conseil départemental de Savoie, service régional de l'Archéologie de la région Rhône-Alpes, Lyon, 148 p.

Remicourt M. (2016) - Évaluation de l'industrie lithique taillée du gisement des Baigneurs, à Charavines (Isère), rapport d'études, musée archéologique de Paladru, service régional de l'Archéologie de la région Rhône-Alpes, Lyon, $14 \mathrm{p}$.

Remicourt M., VAqUer J. (2011) - Aires culturelles et circulations de grandes lames, de plaquettes et de poignards à la fin du Néolithique et au Chalcolithique dans le Midi de la France, in I. Sénépart, T. Perrin, É. Thirault et S. Bonnardin (dir.), Marges, frontières et transgressions : actualités de la recherche, actes des $8^{\text {es }}$ Rencontres méridionales de Préhistoire récente (Marseille, 7-8 novembre 2008), Toulouse, AEP, p. 121-156.

Remicourt M., Bordreuil M. (2012) - Les industries lithiques de la fin du Néolithique dans quelques grottes sépulcrales des garrigues et des piedmonts cévenols du Gard, in M. Sohn et J. Vaquer (dir.), Sépultures collectives et mobiliers funéraires de la fin du Néolithique en Europe occidentale, Toulouse, AEP, p. 213-238.

Renard C.-M. (2003) - Les armatures de flèche de la fin du $I V^{e}$ et du III $I^{e}$ millénaire dans le Bassin parisien, mémoire de DEA, université Paris 1 - Panthéon-Sorbonne, 63 p.

Rey P.-J. (1999) - L'occupation de la Savoie au Néolithique, état des connaissances, mémoire de maîtrise, université de Savoie, Chambéry.

Rey P.-J. (2015) - Aperçu du Néolithique terrestre dans l'avant-pays savoyard et la cluse de Chambéry, Bulles d'archéologies offertes à A. Marguet, Dijon, SAE (Revue archéologique de l'Est, supplément 41), p. 303-385.

Rey P.-J., Marguet A. (2016) - Caractérisation technique et culturelle de la céramique du site lacustre de Conjux 3 (lac du Bourget, Savoie) : le Néolithique final des avant-pays savoyards en question, Bulletin de la Société préhistorique française, 113, 1, p. 57-94.

Roussot-LARroque J. (1984) - Artenac aujourd'hui : pour une nouvelle approche de l'énéolithisation de la France, Revue archéologique du Centre de la France, 23, 2, p. 135-196.

SAINTOT S. (1997) - L'industrie lithique taillée, in P. Pétrequin (dir.), Les sites littoraux de Clairvaux-les-Lacs (Jura), III. Chalain station 3. 3200-2900 av. J.-C., vol. 2, Paris, MSH, p. 371-396.

SAINTOT S. (1998) - Les armatures de flèches en silex de Chalain et de Clairvaux, in P. Pétrequin (dir.), Parures et flèches du Néolithique final à Chalain et à Clairvaux (Jura), une approche culturelle et environnementale (Gallia Préhistoire, 40), p. 204-241.

Saumade H. (1991) - Habitat Néolithique de plein-air de Villeneuve-de-Berg (Ardèche), Ardèche archéologie, 8, p. 14-18.

SAuzade G. (1983) - Les sépultures du Vaucluse, du Néolithique à l'âge du bronze, Paris, LPHP-IPH (Études quaternaires, 6), $251 \mathrm{p}$.

ScImia R., VARÉA S. (1996) - La grotte de la Rouquette (30) Saint-Hilaire-de-Brethmas, au Néolithique final, Alès, GARA, $154 \mathrm{p}$.
SimanjuntaK H.-T. (1998) - Étude de la collection du $D^{r}$ Prunières. Contribution à l'étude de la Préhistoire de la Lozère et des Grands Causses, Banassac, CDPL, 528 p.

Strahm C., Van Willigen S. (2014) - Le concept de culture dans la recherche sur le Néolithique : un regard transfrontalier, in R.-M. Arbogast et A. Greffier-Richard (dir.), Entre archéologie et écologie, une Préhistoire de tous les milieux. Mélanges offerts à Pierre Pétrequin, Besançon, PUFC, p. 467-480.

Thévenot J.-P. (1973) - Le village préhistorique d'Ourouxsur-Saône (résultats des premières fouilles), Mâcon, CRS (Travaux du Centre de recherches de Solutré, 1), $174 \mathrm{p}$.

Thévenot J.-P., Strahm C., Pétrequin P., Bocquet A., Schifferdecker F., Beeching A., Gallay A., Bill J. (1976) - La civilisation Saône-Rhône, Revue archéologique de l'Est et du Centre-Est, 27, 3-4, p. 331-420.

Vaquer J. (1990) - Le Néolithique en Languedoc occidental, Paris, CNRS, $412 \mathrm{p}$.

VAQUER J., BordREUIL M. (2013) - Origine et développement des « pointes de flèches aveyronnaises » dans la région des Grands Causses (France), Préhistoire du Sud-Ouest, 21, 1, p. 63-96.

Voruz J.-L. (1991) - Chronologie du Néolithique d'origine méditerranéenne, Actes du 14e Colloque interrégional sur le Néolithique (Blois, 1987), Blois, SASLV (Bulletin de la Société archéologique, scientifique etlittéraire du Vendômois, supplément), p. 5-33.

Wiessner P. (1983) - Style and Social Informations in Kalahari San Projectile Points, American Antiquity, 48, 2, p. 253-276.

Wilbur C. K. (1990) - Indian Handcrafts, New-York, Globe Pequot Press, $144 \mathrm{p}$.

Winiger A. (2009) - Le mobilier du Néolithique moyen de Saint-Léonard-sur-le-Grand-Pré (Valais, Suisse) : fouilles Sauter 1956-1962, Lausanne, CAR (Cahier d'archéologie romande, 113), $383 \mathrm{p}$.

Maxime Remicourt

UMR 5608 Traces Maison de la Recherche, 5 allées Antonio Machado, 31058 Toulouse cedex 9 m.remicourt@laposte.net

Sylvie SaInTot INRAP Rhône-Alpes-Auvergne, UMR5138 Arar

Centre archéologique de Bron, 12, rue Maggiorini, 69500 Bron sylvie.saintot@inrap.fr

Pierre-Jérôme ReY UMR5204 Edytem Bâtiment Pôle Montagne, 73376 Bourget-le-Lac cedex pierjrey@free.fr 
ANNEXE

\section{TABLEAU D'INVENTAIRE DES ARMATURES À ENCOCHES LATÉRALES EN FRANCE, SUISSE ET ITALIE DU NORD}

\begin{tabular}{|c|c|c|c|c|c|c|c|c|c|}
\hline Pays & Départ./Canton & Commune & Site & Type de site & $\mathbf{N}^{\circ}$ site & $\mathrm{N}^{\circ}$ inv. & Type & Cons. & Références bibliographiques \\
\hline France & Ain & Arbigny & Pont d'Uchizy & station de surface & 108 & 111 & type $5 \mathrm{a}$ & entier & inédit, Duriaud et Barthélémy \\
\hline France & Ain & Châtillon-en-Michaille & En Chatanay & station de surface & 130 & 197 & type $3 a$ & entier & Fillion, 1995 , fig. $6, \mathrm{n}^{\circ} 6$ \\
\hline France & Ain & Pont-d'Ain & Les Combes & station de surface & 033 & 34 & ind & ind. & Combier, 1982 \\
\hline \multirow{2}{*}{ France } & \multirow{2}{*}{ Ain } & \multirow{2}{*}{ Saint-Laurent-sur-Saône } & \multirow{2}{*}{ Bords de la Saône } & \multirow{2}{*}{ station de surface } & 019 & 122 & type $3 a$ & entier & al. 1976 . La Fav $1932, \mathrm{pl}^{41}$ \\
\hline & & & & & 019 & 20 & type $3 b$ & entier & \\
\hline France & Ain & Toussieux & Le Poy at du Chêne & station de surface & 026 & 27 & type $5 b$ & entier & Thévenot et al., 1976 ; Gallay, 1977 \\
\hline France & Ardèche & Bourg-Saint-Andéol & Dolmen du Derbousset & dolmen & 066 & 67 & type $2 \mathrm{a}$ & entier & Saumade, 1993 \\
\hline France & Ardèche & Gras & Dolmen $n^{\circ} 1$ des Clausasses & dolmen & 093 & 96 & type $2 a$ & entier & Bonhomme et Arnal, 1967 \\
\hline France & Ardèche & La Chapelle-sous-Aubenas & RD104 & habitat & 067 & 68 & type $2 a$ & ind. & Durand, 2005 ; étude S. Saintot \\
\hline France & Ardèche & Labeaume & Dolmen du Clos de Jacques & dolmen & 068 & 69 & type 1a & entier & Laforgue, 1978 \\
\hline France & Ardèche & Laurac-en-Vivarais & Le Bois du Talon & habitat & 069 & 70 & type $2 b$ & entier & Evesque et Ribouillault, 1984 \\
\hline France & Ardèche & Le Pouzin & Grotte des Clos & grotte sépulcrale & 095 & 98 & type $3 \mathrm{c}$ & entier & Gély, 1993, pl. 22, fig. 83 \\
\hline France & A tè & 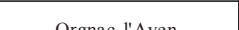 & Lo Roump de Ronze & hohitat & 070 & 126 & type 1a & ind. & Rens. S. Saintot; fouilles R. Gilles \\
\hline France & Ardeche & Orgnac-l Aven & La Baume de Konze & habitat & $0 / 0$ & 71 & type $5 b$ & ind. & Rens. S. Saintot ; fouilles A. Beeching \\
\hline France & Ardèche & Rompon & Grotte de Payre III & grotte sépulcrale & 096 & 99 & type $3 c$ & mésial & Gély, 1993, pl. 24, fig. 93 \\
\hline France & Ardèche & Ruoms & Dolmen du Méandre de Gen & dolmen & 094 & 97 & type $5 b$ & entier & Montj ardin, 1974 , fig. $9, n^{\circ} 10$ \\
\hline France & Ardèche & Saint-Alban-Auriolles & $\begin{array}{c}\text { Le Rochas } \\
\end{array}$ & station de surface & 071 & 72 & type $2 \mathrm{a}$ & entier & Gros, 1999 \\
\hline France & Ardèche & Saint-Marcel-d'Ardèche & Abri de la Vessignée & abri-sous-roche & 072 & 73 & type $1 \mathrm{~b}$ & entier & Beaume, 1996 , fig. $4, n^{\circ} 2$ \\
\hline Frence & A dìn & Caint Moreld" L old th & 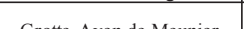 & hbitat & 071 & 147 & type $2 \mathrm{c}$ & entier & Cillos 1089 \\
\hline France & Ardeche & Saint-Marcel-d Ardeche & Grotte-Aven de Meunier & habitat & 0/4 & 215 & type $2 \mathrm{c}$ & entier & Gilles, 1988 \\
\hline France & Ardèche & Saint-Marcel-d'Ardèche & Serre Pluma & habitat & 073 & 74 & type $5 b$ & ind. & Rens. S. Saintot; fouilles R. Gilles \\
\hline France & Ardèche & Saint-Martin-d'Ardèche & Dolmen de la Lone I & dolmen & 123 & 145 & type $5 b$ & entier & inédit, Collections Musée d'Orgnac \\
\hline France & Ardèche & Vallon-Pont-d'Arc & Grotte de Louoï & indéterminé & 075 & 76 & ind & entier & Tscheter, 2002, fig. 12 \\
\hline & & & & & & 77 & type $3 \mathrm{c}$ & mésial & inédit, Collections Musée d'Orgnac \\
\hline France & Ardèche & Villeneuve-de-Berg & Plan des Buns & station de surface & 076 & 146 & type $5 b$ & entier & Saumade, 1991, fig. $5, n^{\circ} 16$ \\
\hline & & & & & & 127 & type $5 \mathrm{~b}$ & proximal & Saumade, 1991, fig. $5, \mathrm{n}^{\circ} 17$ \\
\hline France & Aveyron & Cornus & Dolmen de la Gruelle & dolmen & 087 & 88 & type $5 b$ & entier & Azèmar, 1989, fig. 41 \\
\hline France & Aveyron & Cornus & Dolmen de Prèvinquières & dolmen & 156 & 251 & type $3 \mathrm{c}$ & entier & Azèmar, 1989, fig. 8 \\
\hline France & Avey ron & Creissels & Aven 4 de Bel Air & grotte sépulcrale & 140 & 242 & type $5 a$ & entier & Costantini, 1989 \\
\hline France & Aveyron & Saint-Georges-de-Luzençon & Dolmen de Labro & dolmen & 086 & 87 & type 4 & entier & Azèmar, 1989, fig. 37 \\
\hline France & Avey ron & Saint-Jean-et-Saint-Paul & Dolmen des Agastous & dolmen & 155 & 250 & type $5 b$ & proximal & Azèmar, 1989, fig. 31 \\
\hline France & Aveyron & Sauclières & Comberedonde & station de surface & 088 & 89 & type $3 b$ & mésial & Azèmar, 1989, fig. 58 \\
\hline France & Aveyron & Valady & Dolmen II de Lissalinie & dolmen & 135 & 214 & type $5 b$ & entier & Maury, 1967 \\
\hline France & Bouches-du-Rhône & Martigues & La Couronne & habitat & 138 & 234 & type 1a & entier & Bailloud et Mieg, 1955 , pl. 76 , fig. 19 \\
\hline France & Bouches-du-Rhône & Martigues & Le Collet Redon & habitat & 100 & 103 & type 1a & entier & Courtin, 1974, p. 228-229 \\
\hline France & Charente $\mathrm{C} \rightarrow \mathrm{C}$ & Challignac & Je Camn & habitat & 039 & 40 & type $3 a$ & entier & Fouéré, 1994 \\
\hline & & & & & 039 & 236 & type $5 \mathrm{a}$ & entier & Burnez, 2010, fig. 158 \\
\hline France & Charente-Maritime & Courcoury & Tumulus de Courcoury & tumulus & 035 & 36 & type $5 b$ & entier & Fouéré, 1994, fig. 44, nº 15 \\
\hline & & & & & & 46 & type 1a & entier & Fouéré, 1994, pl. 99, $\mathrm{n}^{\circ} 23$ \\
\hline France & Charente-Maritime & Ecoyeux & Chez Quimand & habitat & 045 & 211 & type 1a & proximal & Fouéré, 1994, pl. 99, nº 19 \\
\hline & & & & & & 212 & type 1a & proximal & Fouéré, 1994, pl. 99, n 22 \\
\hline France & Charente-Maritime & Germignac & & indéterminé & 053 & 54 & type 1a & ind. & Fouéré, 1994 \\
\hline France & Charente-Maritime & Montils & Moulin de Vent & habitat & 051 & 52 & type 1a & ind. & Fouéré, 1994 \\
\hline$F_{0}$ & $C$ lo & Pórimpor & Jounin & bet & 020 & 213 & type 1a & entier & Fouéré, 1994, pl. 82, nº 2 \\
\hline France & Charente-Mlartime & Pengnac & Le Petucinit & nabitat & 034 & 35 & type 1a & entier & Fouéré, 1994 \\
\hline France & Charente-Maritime & Pérignac & Les Fontanies & habitat & 052 & 53 & type 1a & ind. & Fouéré, 1994 \\
\hline France & Charente-Maritime & Plassay & Vallon des Ouchettes & habitat & 041 & 42 & type 1a & proximal & Laporte et Marchand, 2002 \\
\hline France & Charente-Maritime & Rioux & Le Château & habitat & 036 & 37 & type $3 \mathrm{c}$ & entier & Fouéré, 1994, fig. 44 \\
\hline France & Charente-Maritime & Saint-Porchaire & & habitat & 038 & 39 & type 1a & entier & Fouéré, 1994, fig. $44, \mathrm{n}^{\circ} 19$ \\
\hline France & Charente-Maritime & Saint-Seurin-d'Uzet & & indéterminé & 054 & 55 & type 1a & ind. & Fouéré, 1994 \\
\hline France & Charente-Maritime & Semussac & Chez Reine & habitat & 040 & 41 & type 1a & entier & Colle, 1966 ; Fouéré, 1994 \\
\hline France & Charente-Maritime & Tesson & & habitat & 037 & 38 & type 1a & entier & Fouéré, 1994 , fig. $44, \mathrm{n}^{\circ} 18$ \\
\hline France & Côte-d'Or & Barbirey-sur-Ouche & Grotte de Roche-Chèvre & indéterminé & 005 & 5 & ind. & ind. & Joly, 1959 ; Thévenot et al., 1976 \\
\hline France & Côte-d'Or & Seurre & Berges de la Saône & station de surface & 021 & 21 & ind. & ind. & Thévenot et al., 1976 \\
\hline France & Dordogne & Celles & La Blancharderie & habitat & 061 & 62 & type 1a & ind. & Fouéré, 1994 \\
\hline & & & & & & 245 & ind. & proximal & \\
\hline France & Drôme & Buis-les-Baronnies & Grotte de Cost & grotte sépulcrale & 141 & 244 & type $5 b$ & entier & Capitan et Capitan, 1920 \\
\hline & & & & & & 246 & type $5 b$ & proximal & \\
\hline France & Drôme & Die & Beaume Courdeau & grotte sépulcrale & 121 & 130 & type $3 \mathrm{c}$ & \begin{tabular}{|l|} 
entier \\
\end{tabular} & Bernard et al., 1987 \\
\hline France & Drôme & Francillon & Baume Sourde & indétermné & 144 & 249 & type $5 b$ & mésial & Blanc 1957 ; Bill 1973 \\
\hline France & Drôme & La Chapelle-en-Vercors & Les Grands Goulets & station de surface & 132 & 202 & type 1a & ind. & Bocquet, inédit \\
\hline & & & & & & 60 & type $1 \mathrm{~b}$ & entier & \\
\hline & & & & & & 201 & type $2 a$ & entier & \\
\hline & & & & & & 241 & type $2 \mathrm{c}$ & entier & \\
\hline France & Drôme & Mollans-sur-Ouvèze & Hypogée du Perpétairi & hypogée & 059 & 239 & type $2 c$ & entier & Courtin, 1961 \\
\hline & & & & & & 240 & type $2 \mathrm{c}$ & entier & \\
\hline & & & & & & 199 & type $5 b$ & entier & \\
\hline & & & & & & 200 & type $5 b$ & entier & \\
\hline France & Drôme & Montmaur-en-Diois & Grotte du Fournet & grotte sépulcrale & 014 & 14 & type $2 \mathrm{a}$ & entier & Bill, 1973 ; Thévenot et al., 1976 \\
\hline France & Drôme & Mours-Saint-Eusèbe & Ossuaire des Fourneaux & grotte sépulcrale & 091 & 94 & type $2 \mathrm{c}$ & entier & Combier, 1977 ; Beeching, 1980 \\
\hline France & Drôme & Saint-Uze & Gisement II & habitat & 062 & 63 & type 1a & entier & Bocquet et Lagrand, 1976, fig. 15 \\
\hline France & Drôme & Vassieux-en-Vercors & Atelier P51 & station de surface & 092 & 95 & type $2 a$ & ind. & Malenfant, 1976,1979 \\
\hline France & Gard & Durfort & Grotte des Morts & grotte sépulcrale & 139 & 235 & type 1a & proximal & Rem icourt et Bordreuil, 2012 \\
\hline France & Gard & Saint-Hilaire-de-Brethmas & Grotte de la Rouquette & grotte sépulcrale & 049 & 50 & type 1a & entier & Remicourt et Bordreuil, 2012 \\
\hline France & Gironde & Castelnau-de-Médoc & Les Landes & habitat & 055 & 56 & type 1a & ind. & Fouéré, 1994 \\
\hline France & Gironde & Cubzac-les-Ponts & & indéterminé & 057 & 58 & type 1a & ind. & Fouéré, 1994 \\
\hline France & Gironde & Grayan-et-l'Hôpital & La Lède du Gurp & habitat & 042 & 43 & type 1a & entier & Roussot-Larroque, 1984 \\
\hline France & Gironde & Le Porge & & indéterminé & 056 & 57 & type 1a & ind. & Fouéré, 1994 \\
\hline
\end{tabular}




\begin{tabular}{|c|c|c|c|c|c|c|c|c|c|}
\hline Pays & Départ./Canton & Commune & Site & Type de site & $\mathrm{N}^{\circ}$ site & $\mathrm{N}^{\circ}$ inv. & Type & Cons. & Références bibliographiques \\
\hline France & Gironde & Saint-Magne & & indéterminé & 058 & 59 & type 1a & ind. & Fouéré, 1994 \\
\hline France & Haute-Marne & Cohons & La Vergentière & habitat & 115 & 119 & type $3 a$ & mésial & Lepage, 1992 \\
\hline \multirow{2}{*}{ France } & \multirow{2}{*}{ Haute-Savoie } & \multirow{2}{*}{ Annecy } & & & 002 & 75 & type $5 \mathrm{a}$ & proximal & Le Roux, 1908 \\
\hline & & & Station du Port & habitat & 002 & 2 & type $5 b$ & entier & Bailloud et Mieg, 1955, pl. 91, fig. 3 \\
\hline France & Haute-Savoie & Brizon & Plateau de Solaison & station de surface & 119 & 124 & type $5 \mathrm{a}$ & entier & Ginestet, 2001 \\
\hline France & Haute-Savoie & Etrembières & Grotte du Parconnaire & habitat & 111 & 114 & type $2 b$ & entier & inédit, P.-J. Rey \\
\hline France & Haute-Savoie & Musièges & Abri des Douattes & habitat & 048 & 49 & type $5 \mathrm{a}$ & entier & rens. P.-J. Rey, fouilles L. Mevel \\
\hline France & Haute-Vienne & Ambazac & Dolmen de la Lieue & dolmen & 044 & 45 & type $1 b$ & proximal & Dominique, 1961 \\
\hline France & Haute-Vienne & Folles & Dolmen des Goudours & dolmen & 043 & 44 & type $3 \mathrm{c}$ & entier & Crédot et Dominique, 1973 \\
\hline France & Hautes-Alpes & Sigottier & Ferme Robert & habitat & 063 & 64 & type $5 b$ & entier & Bocquet et Lagrand, 1976, fig. 38 \\
\hline France & Hérault & Rouet & Dolmen des Feuilles & dolmen & 135 & 210 & type 1a & entier & Bec Drelon, 2014 \\
\hline France & Hérault & Rouet & Dolmen du Lamalou & dolmen & 060 & 61 & type $5 b$ & entier & Arnal et Louis, 1936 \\
\hline & & & & & & 134 & type 1a & proximal & \\
\hline & & & & & & 139 & type $1 \mathrm{~b}$ & proximal & \\
\hline & & & & & & 143 & type $2 \mathrm{a}$ & entier & \\
\hline & & & & & & 141 & type $2 a$ & proximal & \\
\hline & & & & & & 132 & type $2 a$ & proximal & \\
\hline & & & & & & 136 & type $2 a$ & proximal & \\
\hline & & & & & & 137 & type $3 b$ & entier & \\
\hline France & Isere & Charavines & Les Baıgneurs & habitat & 009 & 133 & type $3 \mathrm{c}$ & entier & Gesler, 2005 \\
\hline & & & & & & 142 & type $3 \mathrm{c}$ & entier & \\
\hline & & & & & & 138 & type $3 \mathrm{c}$ & proximal & \\
\hline & & & & & & 9 & type $3 \mathrm{c}$ & proximal & \\
\hline & & & & & & 140 & type $5 a$ & entier & \\
\hline & & & & & & 135 & type $5 \mathrm{a}$ & entier & \\
\hline & & & & & & 144 & type $5 \mathrm{a}$ & entier & \\
\hline France & Isère & La Balme-les-Grottes & Louvaresse & sépulture & 098 & 101 & type $3 a$ & entier & Thévenot et al., 1976; Gély 1993 \\
\hline France & Isère & Saint-Pierre-de-Chartreuse & Sentier du Col de Belfonds & station de surface & 122 & 131 & type $5 b$ & entier & inédit, rens. C. Bernard \\
\hline France & Isère & Vif & Saint-Loup & indéterminé & 023 & 24 & type $5 b$ & proximal & Thévenot et al., 1976 \\
\hline France & Jura & Brainans & & indéterminé & 006 & 6 & ind. & ind. & Lebrun, 1908 ; Thévenot et al., 1976 \\
\hline & & & & & & 164 & ind. & mésial & Saintot, 1998, pl. $42, \mathrm{n}^{\circ} 158$ \\
\hline & & & & & & 166 & type $3 \mathrm{a}$ & entier & Saintot, 1998, pl. $42, \mathrm{n}^{\circ} 157$ \\
\hline & & & & & & 162 & type $3 \mathrm{a}$ & entier & Saintot, 1998, pl. $43, \mathrm{n}^{\circ} 178$ \\
\hline & & & & & & 163 & type $3 a$ & entier & Saintot, 1998, pl. $43, \mathrm{n}^{\circ} 150$ \\
\hline France & Jura & Clairvaux-les-Lacs & La Motte aux Magnins & habitat & 125 & 161 & type 3a & entier & Saintot, 1998, pl. $43, \mathrm{n}^{\circ} 155$ \\
\hline & & & & & & 172 & type $3 \mathrm{a}$ & proximal & Saintot, 1998, pl. $38, n^{\circ} 84$ \\
\hline & & & & & & 168 & type $3 a$ & proximal & Saintot, 1998, pl. $39, \mathrm{n}^{\circ} 106$ \\
\hline & & & & & & 167 & type $3 c$ & proximal & Saintot, 1998, pl. $40, \mathrm{n}^{\circ} 114$ \\
\hline & & & & & & 165 & type $5 \mathrm{a}$ & entier & Saintot, 1998, pl. $42, n^{\circ} 156$ \\
\hline & & & & & & 8 & type 1a & entier & Thévenot et al., 1976 ; Piningre, 1985 \\
\hline France & Jura & Clairvaux-les-Lacs & Lac de Clairvaux & habitat & 008 & 148 & type $3 a$ & entier & Piningre, 1985 \\
\hline & & & & & & 173 & type $3 c$ & entier & Saintot, 1998, pl. 37, n ${ }^{\circ} 214$ \\
\hline France t & Hurat & Eonten & Chalain 2 & babitat & 126 & 171 & type $3 \mathrm{c}$ & mésial & Saintot, 1998, pl. $39, \mathrm{n}^{\circ} 211$ \\
\hline ( Trance & 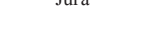 & Fontentu & Chatanit 2 & Ilautat & 120 & 170 & type $3 \mathrm{c}$ & mésial & Saintot, 1998, pl. $39, \mathrm{n}^{\circ} 220$ \\
\hline & & & & & & 169 & type $5 \mathrm{a}$ & distal & Saintot, 1998 , pl. 39, n $^{\circ} 224$ \\
\hline & & & & & & 252 & type $3 \mathrm{a}$ & entier & \\
\hline France & Jura & Fontenu & Chalain 3 & habitat & 157 & 253 & type 3a & entier & Saintot, 1997 \\
\hline & & & & & & 254 & type 4 & entier & \\
\hline & & & & & & 157 & ind. & distal & Piningre, 1985 \\
\hline & & & & & & 151 & type 1a & entier & Thévenot et al., 1976; Piningre, 1985 \\
\hline & & & & & & 153 & type $1 \mathrm{a}$ & entier & \\
\hline & & & & & & 159 & type 1a & entier & \\
\hline & & & & & & 156 & type 1a & proximal & \\
\hline & & & & & & 158 & type $2 \mathrm{a}$ & entier & \\
\hline France & Jura & Fontenu & Lac de Chalain & habitat & 007 & 154 & type $2 a$ & entier & Piningre, 1985 \\
\hline & & & & & & 152 & type $3 \mathrm{a}$ & entier & \\
\hline & & & & & & 150 & type $3 a$ & entier & \\
\hline & & & & & & 160 & type $5 \mathrm{a}$ & entier & \\
\hline & & & & & & 149 & type $5 \mathrm{a}$ & distal & \\
\hline & & & & & & 7 & type $5 \mathrm{a}$ & proximal & \\
\hline & & & & & & 155 & type $5 \mathrm{~b}$ & entier & \\
\hline France & Lot & Marcilhac-sur-Célé & Dolmen de la Devèze & dolmen & 046 & 47 & type 1a & entier & Clottes, 1964 \\
\hline France & Lot & Saint-Cirq-Lapopie & Dolmen de Carbonié & dolmen & 047 & 48 & type $1 \mathrm{a}$ & entier & Clottes, 1964 \\
\hline France & Lozère & Pont-de-Montvert & Cap Français & indéterminé & 109 & 112 & type $2 \mathrm{a}$ & entier & Fages et Paris, 2006 \\
\hline France & Lozère & Pont-de-Montvert & Ravin de la Levade & indéterminé & 110 & 113 & type $5 \mathrm{a}$ & entier & Fages et Paris, 2006 \\
\hline France & Lozère & Saint-Rome-de-Dolan & Dolmen de La Lozère & dolmen & 084 & 85 & type 1a & entier & Simanjuntak, 1998, fig. 43 \\
\hline France & Lozère & Saint-Rome-de-Dolan & Grotte d'Almières & grotte sépulcrale & 085 & 86 & type 1a & proximal & Simanjuntak, 1998, fig. 45 \\
\hline France & Lozère & Sainte-Enimie & Tumulus du Devez Viel & tumulus & 065 & 66 & type $1 b$ & entier & Fages et Vacquier, 2007 \\
\hline France & Nièvre & Saint-Martin-d'Heuille & & station de surface & 032 & 33 & ind. & ind. & Thévenot, 1985 \\
\hline France & Puy-de-Dôme & Saint-Anthème & Vialvieille & indéterminé & 081 & 82 & type $1 b$ & entier & Georges, 2007, pl. 51 \\
\hline France & Puy-de-Dôme & Valcivières & Balay oux & indéterminé & 083 & 84 & type $5 \mathrm{a}$ & entier & Georges, 2007, pl. $52, \mathrm{n}^{\circ} 2$ \\
\hline France & Rhône & Lyon & Rue Auguste Isaac & habitat & 077 & 78 & type $5 \mathrm{a}$ & ind. & Etude S. Saintot, fouilles J.-F. Treffort \\
\hline France & Rhône & Odenas & & station de surface & 131 & 217 & ind. & \begin{tabular}{|l|} 
distal \\
\end{tabular} & Sayoye 1898 \\
\hline & & Vaenas & & & 101 & 198 & type $3 \mathrm{a}$ & proximal & Savoye, 1098 \\
\hline France & Rhône & Quincieux & Grange Rouge & habitat & 079 & 80 & type $2 a$ & ind. & Etude S. Saintot, fouilles J. Ramponi \\
\hline France & Rhône & Quincieux & Le Grand Veyssieux & indéterminé & 025 & 26 & ind. & ind. & Thévenot et al., 1976 \\
\hline France & Rhône & Quincieux & Les Forgettes & habitat & 078 & 79 & type $2 a$ & ind. & Etude S. Saintot; Motte, 2015 \\
\hline France & Rhône & Saint-Priest & Les Feuilly & habitat & 080 & 81 & type $2 \mathrm{a}$ & ind. & Etude S. Saintot, fouilles Henon \\
\hline France & Saône-et-Loire & Digoin & Le Volgu & habitat & 011 & 11 & ind. & ind. & Thévenot et al., 1976 \\
\hline France & Saône-et-Loire & Lacrost & Les Varennes & station de surface & 112 & 115 & ind. & ind. & Renseignement P.-J. Rey \\
\hline France & Saône-et-Loire & Le Villars & & station de surface & 118 & 123 & type $5 \mathrm{a}$ & entier & La Fay, 1932, pl. 41 \\
\hline France & Saône-et-Loire & Mâcon & Berges de la Saône & station de surface & 136 & 218 & type $1 b$ & entier & Ferry, 1870 \\
\hline
\end{tabular}




\begin{tabular}{|c|c|c|c|c|c|c|c|c|c|}
\hline Pays & Départ./Canton & Commune & Site & Type de site & $\mathrm{N}^{\circ}$ site & $N^{\circ}$ inv. & Type & Cons. & Références bibliographiques \\
\hline \multirow{15}{*}{ France } & \multirow{15}{*}{ Saône-et-Loire } & \multirow{15}{*}{ Ouroux-sur-Saône } & \multirow{15}{*}{ Le Grand Bois / Le Taillis } & \multirow{15}{*}{ habitat } & \multirow{15}{*}{016} & 228 & type 1a & entier & \multirow{2}{*}{ Thévenot, 1973, fig. 36} \\
\hline & & & & & & 227 & type $1 \mathrm{a}$ & entier & \\
\hline & & & & & & 230 & type $1 \mathrm{~b}$ & entier & Thévenot, 1973, fig. 75 \\
\hline & & & & & & 226 & type $1 b$ & proximal & Thévenot, 1973, fig. 36 \\
\hline & & & & & & 233 & type $1 b$ & proximal & \\
\hline & & & & & & 229 & type $2 \mathrm{a}$ & entier & Thévenot, 1973, fig. 75 \\
\hline & & & & & & 220 & type $3 a$ & entier & \\
\hline & & & & & & 223 & type $3 c$ & entier & Thévenot, 1973, fig. 36 \\
\hline & & & & & & 224 & type $3 c$ & entier & \\
\hline & & & & & & 231 & type $3 c$ & entier & Thévenot, 1973, fig. 75 \\
\hline & & & & & & 17 & type $3 c$ & distal & \\
\hline & & & & & & 225 & type $3 c$ & mésial & Thévenot, 1973, fig. 36 \\
\hline & & & & & & 232 & type $3 c$ & proximal & Thévenot, 1973, fig. 75 \\
\hline & & & & & & 222 & type $3 c$ & proximal & \\
\hline & & & & & & 221 & type $3 c$ & proximal & Thevenot, 19/3, 11g. 36 \\
\hline France & Saône-et-Loire & Prety & Berges de la Saône & station de surface & 017 & 18 & ind. & \begin{tabular}{|c|} 
ind. \\
\end{tabular} & Thévenot et al., 1976 \\
\hline France & Saône-et-Loire & Sennece-les-Macon & La Sénétrière & station de surface & 022 & 23 & type $3 a$ & entier & La Fay, 1932, pl. 41 ; Thévenot et al., 1976 \\
\hline France & Saône-et-Loire & Simandre & Les Crozes & indéterminé & 114 & 117 & type $5 \mathrm{a}$ & entier & Duriaud, 1999 \\
\hline France & Saône-et-Loire & Tournus & La Croix Léonard & indéterminé & 113 & 116 & type $3 a$ & entier & Duriaud, 1999 \\
\hline France & Saône-et-Loire & Uchizy & La Saône & station de surface & 020 & 22 & type $1 \mathrm{~b}$ & entier & La Fay, 1932, pl. 41 ; Thévenot et al., 1976 \\
\hline France & Saône-et-Loire & Vergisson & La Roche & station de surface & 137 & 219 & type $2 a$ & entier & Ferry, 1870 \\
\hline & & & & & & 177 & type $2 \mathrm{a}$ & entier & Thévenot et al., 1976; Rey, 1999 \\
\hline France & Savoie & Aiguebelette-le-Lac & Boffard & habitat & 127 & 176 & type $2 b$ & entier & Thévenot et al. 1976 ; Rey, 1999 \\
\hline & & & & & & 186 & ind. & mésial & Remicourt, 2015 \\
\hline & & & & & & 175 & type $2 b$ & entier & Thévenot et al. 1976; Rey, 1999 \\
\hline & & & & & & 178 & type $3 c$ & entier & \\
\hline & & & & & & 1 & type $5 \mathrm{a}$ & entier & \\
\hline France & Savoie & Alguebelette-le-Lac & Lac d'Aiguebelette & habitat & 001 & 174 & type $5 \mathrm{a}$ & entier & Rey, 1999 \\
\hline & & & & & & 180 & type $5 \mathrm{a}$ & mésial & \\
\hline & & & & & & 179 & type $5 b$ & entier & \\
\hline & & & & & & 190 & type 3a & entier & \\
\hline & & & & & & 189 & type $3 a$ & entier & \\
\hline & & & & & & 182 & type $3 a$ & entier & \\
\hline & & & & & & 192 & type 3a & entier & \\
\hline France & Savoie & & & & & 191 & type 3a & entier & \\
\hline France & Savole & Aiguebelette-le-Lac & Les Roseaux / Boffard? & habitat & 128 & 184 & type $5 \mathrm{a}$ & entier & Rey, 1999 ; Rem icourt, 2015 \\
\hline & & & & & & 187 & type $5 a$ & entier & \\
\hline & & & & & & 188 & type $5 \mathrm{a}$ & mésial & \\
\hline & & & & & & 185 & type $5 \mathrm{a}$ & proximal & \\
\hline & & & & & & 181 & type $5 b$ & entier & \\
\hline & & & & & & 204 & ind. & ind. & \\
\hline France & Savoie & Bozel & Le Chenet des Pierres & habitat & 133 & 203 & type $2 b$ & entier & inédit, fouilles P.-J. Rey \\
\hline France & Savoie & Conjux & Les Côtes & habitat & 120 & 125 & type $5 b$ & entier & inédit, fouilles A. Marguet \\
\hline France & Vaucluse & Bonnieux & station des Fabbri & habitat & 101 & 104 & type $1 \mathrm{a}$ & ind. & Courtin, 1974 \\
\hline France & Vaucluse & Ménerbes & Dolmen de la Pichone & dolmen & 117 & 121 & type $3 a$ & entier & Sauzade, 1983 , fig. 76 \\
\hline France & Vaucluse & Robion & Grotte du Jas de Juvert & habitat & 099 & 102 & type $1 \mathrm{a}$ & ind. & Courtin, 1974, p. 257 \\
\hline France & Vaucluse & Venasque & Grotte du Stade & grotte sépulcrale & 031 & 32 & ind. & mésial & Sauzade, 1978,1983 \\
\hline Italie & Turin & Orrido & Chianocco & habitat & 104 & 107 & type $5 b$ & entier & Bertone, 1987 \\
\hline Italie & Verone & Bardolino & palafitte de Porto Cisano & habitat & 142 & 247 & type $3 \mathrm{c}$ & entier & Baioni et al., 2015 \\
\hline Suisse & Berne & Lüscherz & Dorfstation & habitat & 013 & 13 & ind. & ind. & Thévenot et al., 1976 \\
\hline Suisse & Berne & Sutz-Latringen & Sutz-Latringen & habitat & 050 & 51 & type $1 \mathrm{~b}$ & proximal & Mallet, 1992, pl. 66 \\
\hline & & & & & & 195 & ind. & \begin{tabular}{|c|} 
ind. \\
\end{tabular} & \\
\hline Suisse & Fribourg & Dellev & Portalban 2 & habitat & 028 & 29 & type $2 \mathrm{a}$ & entier & Honegger 2001 \\
\hline Sulsse & Fribourg & Delley & Portalban 2 & habitat & 028 & 194 & type $3 c$ & entier & Honegger, 2001 \\
\hline & & & & & & 196 & type $5 \mathrm{a}$ & distal & \\
\hline Suisse & Fribourg & Frasses & Prazdu Doux & habitat & 105 & 108 & type $5 b$ & entier & Mauvilly et Ruffieux, 2008 \\
\hline Suisse & Fribourg & La Combaz & Chables & habitat & 107 & 110 & type $5 \mathrm{a}$ & proximal & Anderson, 2008 \\
\hline Suisse & Fribourg & La Faye & Lully & habitat & 106 & 109 & type $5 \mathrm{a}$ & proximal & Agustoni et al., 2008 \\
\hline Suisse & Fribourg & Mur-Chenevières & Guévaux I & habitat & 015 & 16 & ind. & ind. & Thévenot et al., 1976 \\
\hline Suisse & Genève & Corsier & Le Port ou La Gabiule & habitat & 143 & 248 & type $3 a$ & mésial & Gallay et Corboud, 1979 \\
\hline Suisse & Genève & Genève & Les Eaux-Vives & habitat & 012 & 12 & ind. & ind. & Thévenot et al., 1976 \\
\hline & & & & & & 105 & type $3 c$ & proximal & \\
\hline Suisse & Genève & Genève & Parc Lagrange & habitat & 102 & 129 & type $5 \mathrm{a}$ & entier & Pugin et Corboud, 2006 \\
\hline Suisse & Jura & Alle & Bois Noir & habitat & 103 & 106 & ind. & ind. & Archéologie Suisse 37, 2014 \\
\hline Suisse & Neuchâtel & Auvernier & Brise Lame & habitat & 003 & 3 & ind. & ind. & Thévenot et al., 1976 \\
\hline Suisse & Neuchâtel & Saint-Blaise & Bain des Dames & habitat & 018 & 19 & type $3 \mathrm{a}$ & entier & Honegger, 2001 \\
\hline & & & & & & 206 & type $1 \mathrm{a}$ & entier & Winiger, 2009, pl. $25, \mathrm{n}^{\circ} 9$ \\
\hline & & & & & & 207 & type 1a & entier & Winiger, 2009, pl. 19, $\mathrm{n}^{\circ} 149$ \\
\hline Suisse & Valais & Saint-Léonard & Sur le Grand Pré & habitat & 134 & 208 & type $2 b$ & entier & Winiger, $2009, \mathrm{pl} .1, \mathrm{n}^{\circ} 12$ \\
\hline & & & & & & 209 & type $2 b$ & mésial & Winiger, 2009, pl. $5, \mathrm{n}^{\circ} 175$ \\
\hline Suisse & Valais & Sion & Dolmen MXII & dolmen & 129 & 205 & type 1a & entier & Favre et Mottet, 1990 \\
\hline Suisse & Vaud & Concise & & habitat & 010 & 10 & ind. & ind. & Thévenot et al., 1976 \\
\hline Suisse & Vaud & Morges Tolochenaz & La Poudrière & habitat & 030 & 31 & type $5 \mathrm{a}$ & entier & Francillon et Gallay, 1978 ; Picard, 1979 \\
\hline Suisse & Vaud & Yverdon & Avenue des Sports & habitat & 029 & 30 & type $5 \mathrm{a}$ & entier & Uerpmann, 1976; Honegger, 2001 \\
\hline
\end{tabular}

